\title{
Multi-Exposed recordings for 3D Lagrangian Particle Tracking with Multi-Pulse Shake-The-Box
}

\author{
Matteo Novara, Daniel Schanz, Reinhard Geisler, Sebastian Gesemann, Christina \\ Voss, Andreas Schröder \\ German Aerospace Center (DLR), Institute of Aerodynamics and Flow Technology \\ Bunsenstrasse 10, 37073 Göttingen, Germany \\ Corresponding author: matteo.novara@dlr.de, Tel. +495517092995
}

\begin{abstract}
The recent introduction of the Multi-Pulse Shake-The-Box (MP-STB) method opened the possibility of extending 3D Lagrangian particle tracking (LPT) to the investigation of high-speed flows, where long time-resolved sequences of recordings are currently not available due to the limited acquisition frequency of high-speed systems. The MPSTB technique makes use of an iterative approach to overcome the limitations posed by the short observation time offered by a multi-pulse recording sequence. Multi-pulse sequences are typically obtained by synchronizing multiple illumination systems in order to generate bursts of laser pulses where the time separation can be freely adjusted down to less than a microsecond. Several strategies can be adopted for the recording of multi-pulse sequences; a dual camera system can be adopted in order to separate the single pulses onto the camera frames (either by means of polarization or timing), while the use of multi-exposed frames allows for the employment of a single imaging system, largely reducing the complexity and cost of the experimental setup.

The main strategies to generate multi-pulse recording sequences are presented here; the application and performances of the MP-STB method are discussed based on the analysis of experimental data from the investigation of three turbulent boundary layer flows at velocities ranging from 10 to approximately $30 \mathrm{~m} / \mathrm{s}$. Results show the capability of the MP-STB technique in reconstructing accurate track fields which can be exploited both to describe instantaneous flow structures and to produce highly spatially resolved statistics by means of ensemble average in small bins. The iterative reconstruction and tracking strategy for MP-STB can be successfully adapted to the case of multi-exposed frames. Results suggest that, despite the increase in particle image density resulting from the double-exposed particle images, the adoption of multi-exposed recordings has the potential to become the technique of choice for the recording of multi-pulse sequences suitable for Lagrangian particle tracking in high-speed flows.
\end{abstract}

\section{Introduction}

Lagrangian particle tracking allows for the accurate measurement of single particle tracers' position, velocity and acceleration. The scattered information obtained from particle tracking can be accurately interpolated onto a regular grid by means of data assimilation algorithms (FlowFit - Gesemann et al 2016, Vortex-in-cell+ - Schneiders and 
Scarano 2016) providing access to the spatial gradients and, given the accurate material acceleration measurement by particle tracks, to instantaneous 3D pressure fields (Van Oudheusden 2013, Van Gent et al 2017, Huhn et al 2018).

The Shake-The-Box technique (STB, Schanz et al 2016) enables the reconstruction of single particle tracks in 3D over long time-resolved sequences of recordings from a multi-camera imaging system. The STB method makes use of the Iterative Particle Reconstruction (IPR, Wieneke 2013). An initial phase where tracks are identified over the first few realizations is followed by the prediction of the particle location at subsequent recordings; the predicted location is then corrected by means of an image matching scheme (shaking step). Thanks to the iterative approach of the IPR and to the exploitation of the time information from multiple recordings (Hain and Kähler 2007, Novara et al 2010, Sciacchitano et al 2012, , Lynch and Scarano 2013 concerning correlation-based techniques; Cierpka et al 2013 for particle tracking), the STB is able to cope with unprecedented seeding concentration levels when compared to standard 3D LPT techniques based on single-pass 2D-particle detection and 3D-triangulation (e.g. 3D-PTV, Maas et al 1993), and comparable to those typically applied for tomographic-PIV (Tomo-PIV, Elsinga et al 2006), even when adopting advanced processing methods (Lynch and Scarano 2015). Schanz et al (2016) showed that STB is able to accurately reconstruct particle tracks nearly free of ghost particles (Elsinga et al 2011) at particle image densities exceeding 0.1 ppp (particles-per-pixel), Huhn et al (2017).

Time-resolved STB applications have been limited to relatively low flow velocities, typically below $40 \mathrm{~m} / \mathrm{s}$, depending on the available hardware components. On the other hand, most industrial and aerodynamics applications require higher velocities, where, due to the current limitations in terms of recording frequency, the acquisition of long time-resolved sequences of recordings suitable for STB processing would result in significant compromises in terms of the spatial and dynamic range of the measurement (e.g. limited size of the field of view, large particle displacements, low image signal-to-noise ratio). In these conditions, multi-pulse systems can be employed, which make use of dual double-frame illumination systems, synchronized to produce short sequences of (typically four) time-resolved pulses (Schröder et al 2013, Lynch and Scarano 2014, Blinde et al 2015).

The STB technique has been recently extended to the case of multi-pulse data (Novara et al 2016a); an iterative strategy based on the sequential application of IPR and particle tracking is employed to progressively reduce the complexity of the reconstruction/tracking problem (i.e. particle image density) and increase the number of successfully reconstructed tracks, Figure 1.The performances of this novel strategy have been demonstrated by means of synthetic experiments (Novara et al 2016b) and the technique has been successfully applied to the investigation of turbulent boundary layers (Novara et al 2016a), flow over a laminar wing (Geisler et al 2016) and to data from a subsonic jet experiment at Mach 0.84 (Manovski et al 2016).

Several strategies can be adopted for the recording of multi-pulse sequences; due to the long exposure time of the second frame when cameras are operated in double-shutter mode, multiple camera systems can be installed in order to separate the pulses onto the camera frames and avoid multi-exposed recordings. Lynch and Scarano (2014) proposed the use of three independent camera systems, the first two operating in single-frame mode to acquire the first two pulses and the third one operating in double-frame mode to capture the last two pulses. The main limitation of this approach lies in the large number of cameras required (at least nine); furthermore, the use of three 


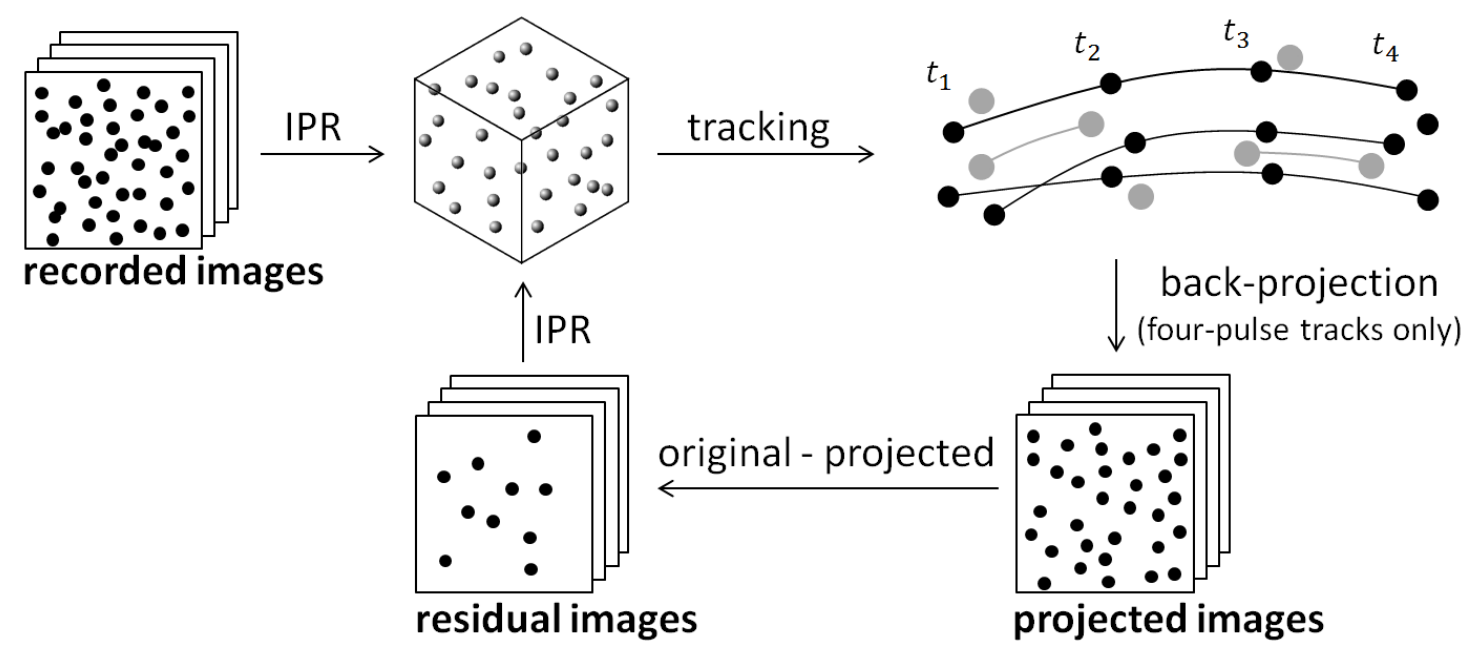

Figure 1. Iterative processing strategy for MP-STB; the IPR reconstruction of recorded/residual images, the tracking step and the evaluation of back-projected and residual images constitute a single MP-STB iteration. Complete four-pulse tracks are indicated in black, incomplete shorter tracks in gray.

independent imaging systems prevents the a-posteriori identification and correction of the camera displacements due to structural vibrations, a situation often encountered in large industrial facilities (Novara et al 2016a).

The use of two independent imaging systems has been proposed by Kähler and Kompenhans (2000) and adapted by Schröder et al (2013); two double cavity lasers emit two pulses each at orthogonal polarization directions and camera lenses are equipped with polarization filters to separate the four pulses on the different camera frames. This technique is referred to as polarization-based strategy in the remainder of the paper, and it has already been employed in combination with MP-STB (Novara et al 2016a, Geisler et al 2016, Manovski et al 2016). On the other hand, the introduction of framing-optimized exposure cameras (FOX, Geisler 2014) capable of acquiring two frames with short exposure time - at the expense of halving the sensor resolution along one direction - made it possible to implement an acquisition strategy where the pulse separation is based solely on timing (timing-based strategy).

The advantages and criticalities of these pulse separation strategies are discussed in the present study; the two approaches are applied to the same boundary layer flow at $10 \mathrm{~m} / \mathrm{s}$ and results are assessed in terms of percentage of successfully reconstructed tracks and compared to data from direct numerical simulation (DNS).

Due to restrictions in terms of availability of cameras and/or optical access to the test section (particularly limited in many industrial facilities), the use of six or eight cameras required to build two independent 3D imaging systems might not be possible. In order to ease on this limitation and to simplify the experimental setup of MP-STB investigations, an alternative acquisition strategy based on multi-exposed frames is proposed here, where the first two laser pulses are recorded on the first frame and the remaining two pulses on the second frame. The application of the Multi-Pulse STB method to double-exposed recordings is assessed based on the analysis of instantaneous recordings from a turbulent boundary layer investigation at $15 \mathrm{~m} / \mathrm{s}$ and its performances compared to those achieved adopting the aforementioned pulse separation strategies. Furthermore, the MP-STB is applied to multiexposed recordings from the investigation of a turbulent boundary layer flow with adverse pressure gradient at 


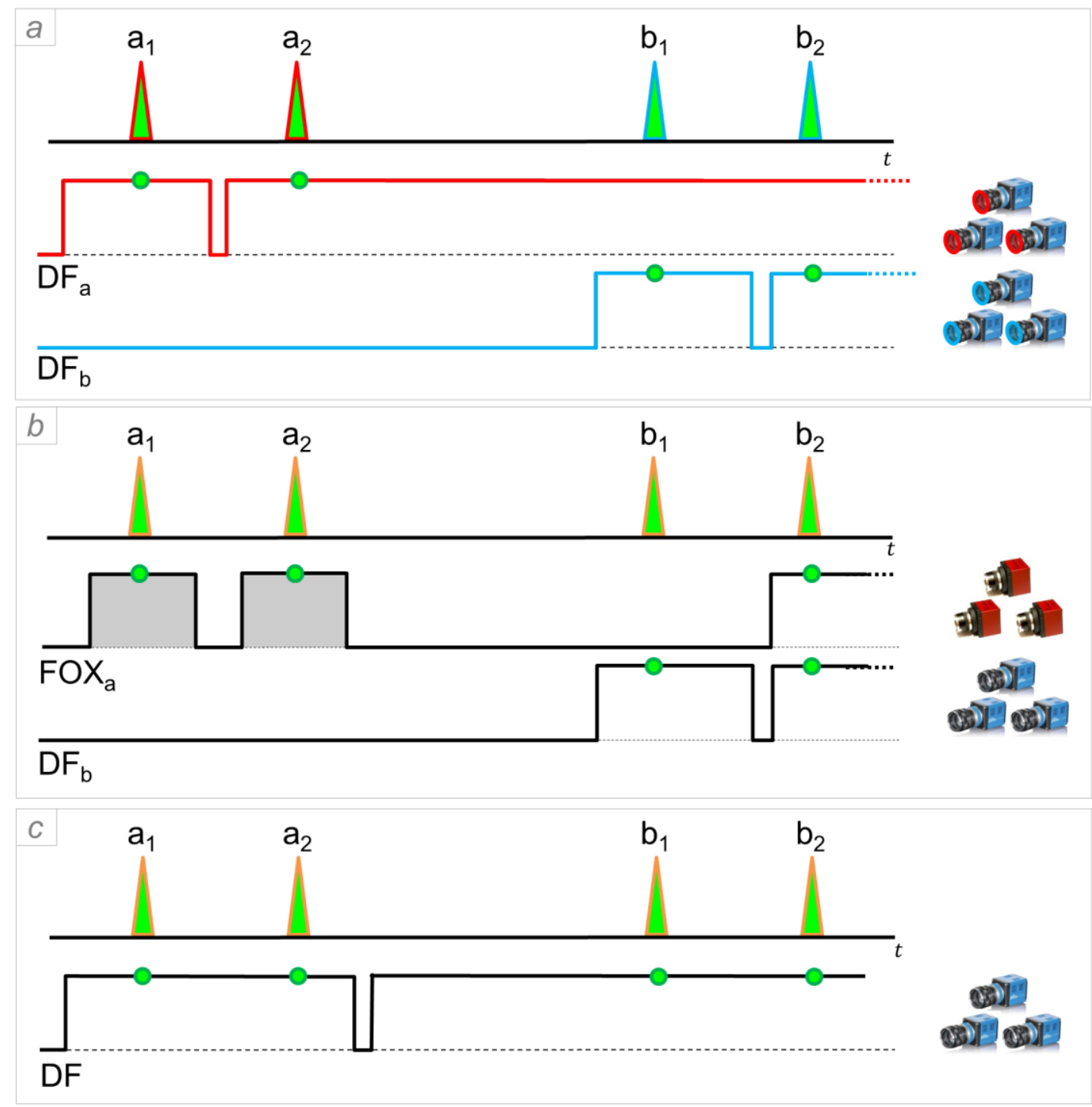

Figure 2. Pulse separation timing diagrams for multi-pulse acquisition strategies; red, blue and orange indicate horizontal, vertical and circular polarization respectively, $a$ and $b$ refer to the two illumination and imaging systems, 1 and 2 to the first and second laser pulse. $a$ : polarization-based strategy, $b$ : timing-based strategy (gray areas indicate FOX frames at reduced resolution), $c$ : multi-exposed strategy.

$29.25 \mathrm{~m} / \mathrm{s}$, where long sequences of statistically independent realizations allowed for the evaluation of the boundary layer profiles. The availability of planar PIV data from the same experiment provides a term of comparison for the MP-STB results. The experimental conditions and setup are described here together with the main processing parameters for the MP-STB processing.

\section{Recording strategies for multi-pulse sequences}

Three strategies to record particle images illuminated by four laser pulses separated by a few microseconds are proposed. The first two techniques, namely the polarization- and timing-based pulse separation strategies, aim at the 
realization of single exposed images by adopting a dual imaging system of cameras operating in double-shutter mode. On the other hand, the third strategy is based on a single 3D imaging system, where the four laser pulses are recorded double-exposing each of the two camera frames.

When cameras are operated in double-shutter mode the read-out operation of the first frame results in a long exposure time (approximately 20 milliseconds) for the second frame; as a consequence, when a multi-pulse sequence of four laser pulses separated by a few microseconds is recorded, multi-exposed particle images are generated for the second frame. The polarization-based pulse separation technique is illustrated in Figure 2a. In this example, the two imaging systems consist of three dual-frame (DF) cameras each; lasers $a$ and $b$ emit light with horizontal and vertical polarizations respectively and pulses are separated by applying polarization filters to the camera lenses (indicated by red and blue circles in Figure 2a). The adoption of polarized light imposes restrictions in terms of particle tracers (i.e. spherical shape) and viewing angles (typically limited to $20^{\circ}$ with respect to the forward and backward scattering plane) in order to preserve the polarization of scattered light. Furthermore, the use of polarizing filters and the different viewing angles with respect to the Mie scattering lobes can result in strong differences in terms of particle peak intensities within the two systems, affecting the STB tracking scheme and potentially leading to loss of particles (Novara et al 2016a). To avoid these restrictions, a pulse separation strategy based solely on timing can be adopted, Figure $2 b$; framing-optimized exposure cameras, capable of recording two short-exposure frames in rapid succession, are used to replace the DF cameras for one of the two imaging systems $\left(F O X_{a}\right)$. The FOX cameras exploit a special readout system for off-the-shelf image sensors, where the first two images are obtained from binned pixel lines of the sensor (even and odd lines added and frame 1 and 2 stored onchip in a line-interleaved pattern) using the electronic shutter to limit the exposure time (Geisler 2014); as a consequence, the images related to the first two pulses in the sequence have reduced resolution (by a factor two) along one direction of the sensor, but no polarizing filter is necessary to avoid multi-exposed images. The laser illumination is therefore switched from linear to circular polarization. Furthermore, the FOX cameras deliver a third frame which can be used to record the last pulse(s) of the multi-pulse sequence; this additional frame could be used to re-align the two imaging systems in case of displacements due to structural vibrations.

Both the polarization- and timing-based techniques require two 3D imaging systems consisting each of three or more cameras; in order to reduce the complexity of the experimental setup, an acquisition strategy based on multiexposed frames is proposed here, where a single 3D imaging system is adopted. This solution also represents an advantage considering the typically limited optical access of industrial facilities. The timing diagram for the multiexposed acquisition strategy is presented in Figure 2c. Circular polarization for all four laser pulses is adopted; the first two pulses are recorded on the first camera frame while the last two pulses are imaged on the second frame.

The performances of the Multi-Pulse STB in terms of percentage of successfully reconstructed particle tracks are assessed for the aforementioned recording strategies based on the analysis of experimental data from turbulent boundary layer investigations at 10 (polarization- and timing-based pulse separation) and $15 \mathrm{~m} / \mathrm{s}$ (multi-exposed recordings). Results are presented in the following sections together with the description of the experimental setups. 
Figure 3. Experimental setup in the $1 \mathrm{~m}-$ Wind Tunnel at the DLR Göttingen (top/bottom: views from both sides of the test section).

The timing diagram of the four-pulse sequences is also shown (bottom).
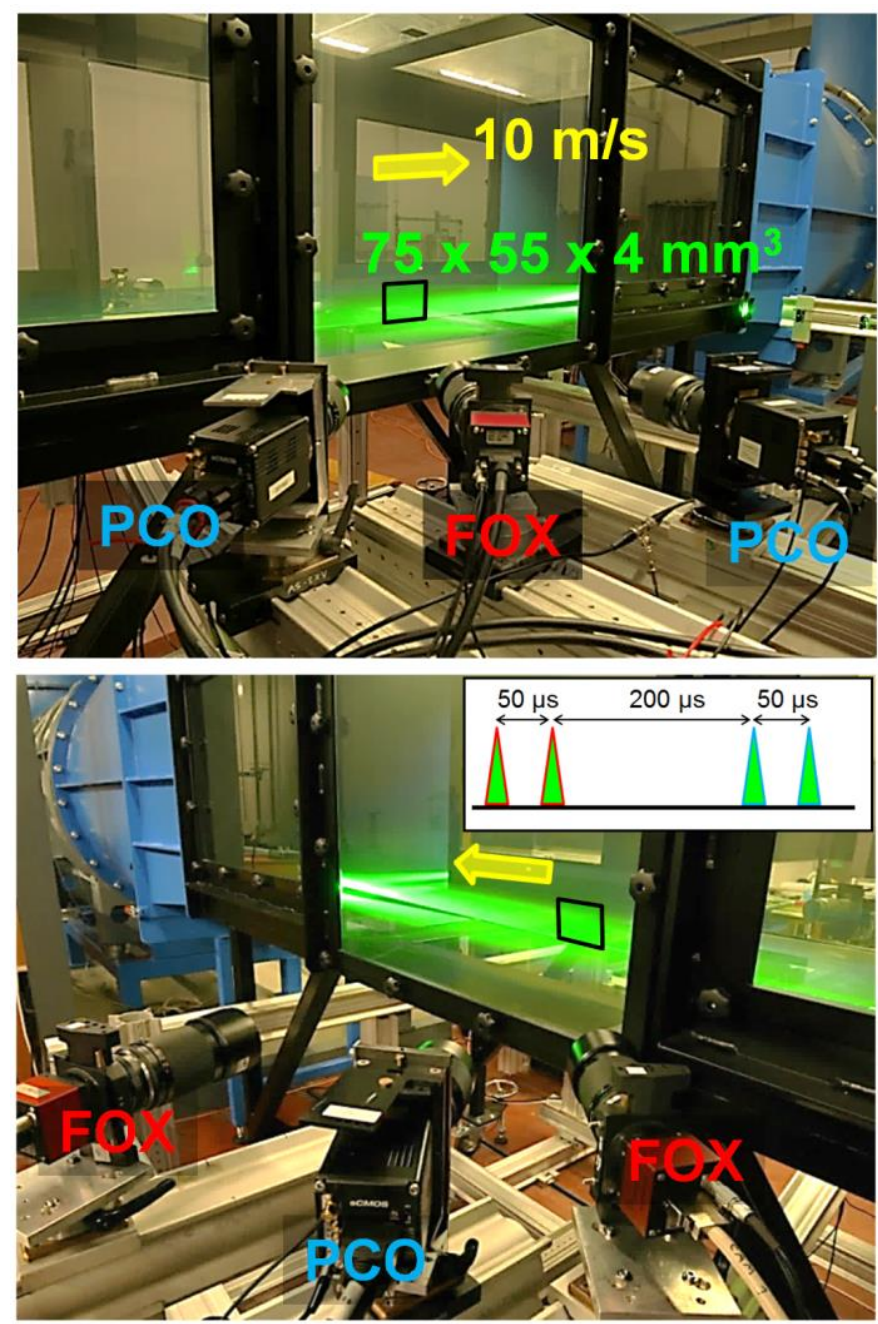

\section{Experimental assessment of pulse separation recording strategies}

In order to assess the effect of the two different pulse-separation strategies on the Multi-Pulse STB results, an experiment has been designed where a turbulent boundary layer at $10 \mathrm{~m} / \mathrm{s}$ is investigated using both PCO Edge sCMOS and FOX cameras; since the latter can be operated in dual-frame mode and in FOX-mode (as shown in the timing diagram in Figure 2b), both the polarization- and timing-based strategies can be implemented.

The experiment was carried out in the $1 \mathrm{~m}$-Wind Tunnel at DLR Göttingen. Illumination was provided by a pair of double-cavity BigSky Ultra 400 lasers and the flow seeded with DEHS (Di-Ethyl-Hexyl-Sebacate) droplets $(\approx 1 \mu \mathrm{m}$ diameter). A $75 \times 55 \times 4 \mathrm{~mm}^{3}$ volume (in stream-wise, wall-normal and span-wise directions respectively) was illuminated and imaged by three FOX cameras $(2048 \times 2048 p x, 7.5 \mu \mathrm{m}$ pixel size $)$ and three PCO Edge sCMOS cameras $\left(2560 \times 2160 p x, 6.5 \mu \mathrm{m}\right.$ pixel size) composing the $F O X_{a}$ and $D F_{b}$ systems respectively, Figure 3 . When operated in FOX-mode, the Frame Optimized cameras acquire the first two frames at reduced (vertical) resolution and thus the raw images have a pixel aspect ratio of 1:2. The digital resolution was approximately $35 \mathrm{px} / \mathrm{mm}$. An uneven pulse separation was chosen in order to increase the dynamic range of the measurement (Novara et al 2016b); a time separation of $50 \mu s$ was set between the first and last two pulses, while a larger separation of $200 \mu s$ 


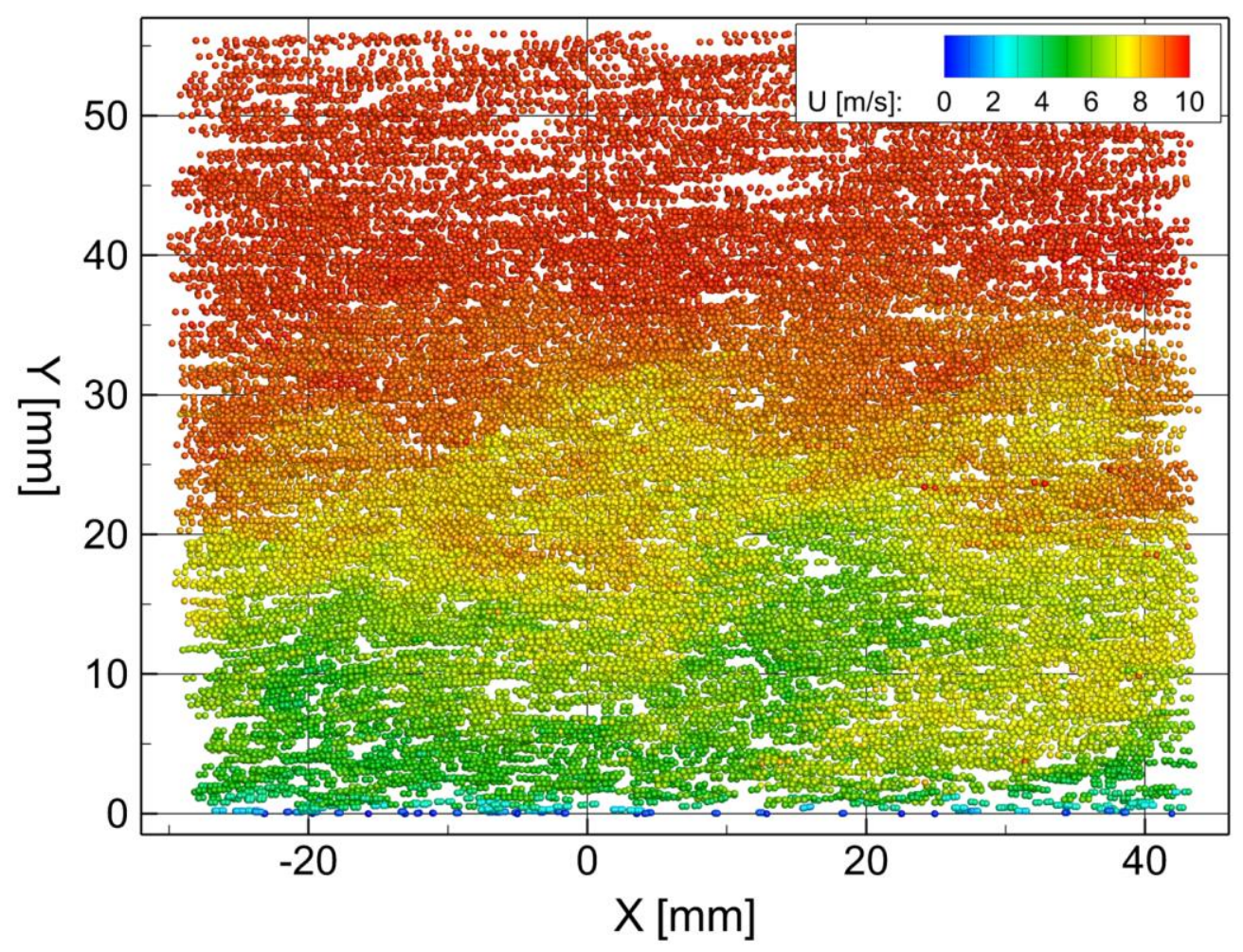

Figure 4. Instantaneous MP-STB result (polarization-based strategy); approximately 10,000 four-pulse tracks color-coded by the stream-wise velocity component.

was chosen between pulses two and three. A sequence of 10,000 four-pulse recordings was acquired at a frequency of $10 \mathrm{~Hz}$ in order to provide statistical convergence and allow for the evaluation of flow statistics.

A relatively low particle image density was used to guarantee optimal reconstruction and tracking performances, therefore allowing the assessment of the sole effect of the pulse separation strategy. The particle image density is computed as the number of particle images identified within the active area of the sensor divided by the size of the same area in pixels, and it is measured in particles-per-pixel ( $p p p)$. As a consequence of the different viewing directions, due to the different path of the lines-of-sight across the illuminated volume and their angle with respect to the Mie scattering lobes, different image density values are typically found for the several cameras; for the present case, an average of approximately $0.015 p p p$ was estimated across the imaging system. At low image density levels the number of actual particles images can be accurately determined by means of intensity local maxima identification; besides the few particles leaving the illuminated volume within the four-pulse sequence, this number corresponds to the number of actual four-pulse tracks that can be reconstructed within the investigated volume. As a consequence, the percentage of successfully retrieved tracks can be accurately estimated for the two strategies.

Details regarding the MP-STB processing strategy can be found in Novara et al (2016a and 2016b); three MP-STB iterations have been performed and approximately $10,000 \div 20,000$ instantaneous tracks are found depending on the particle image density and the recording strategy. An instantaneous MP-STB result is shown in Figure 4, where the location of the tracked particles is marked, and color-coded based on the stream-wise velocity component. 
Figure 5. Mean profiles of normalized turbulent fluctuation intensities along the wall normal direction; results from STB shown with colored symbols, DNS result shown with black curves. Top: STB result from the polarization-based pulse separation strategy. Bottom: STB result from the timing-based strategy.

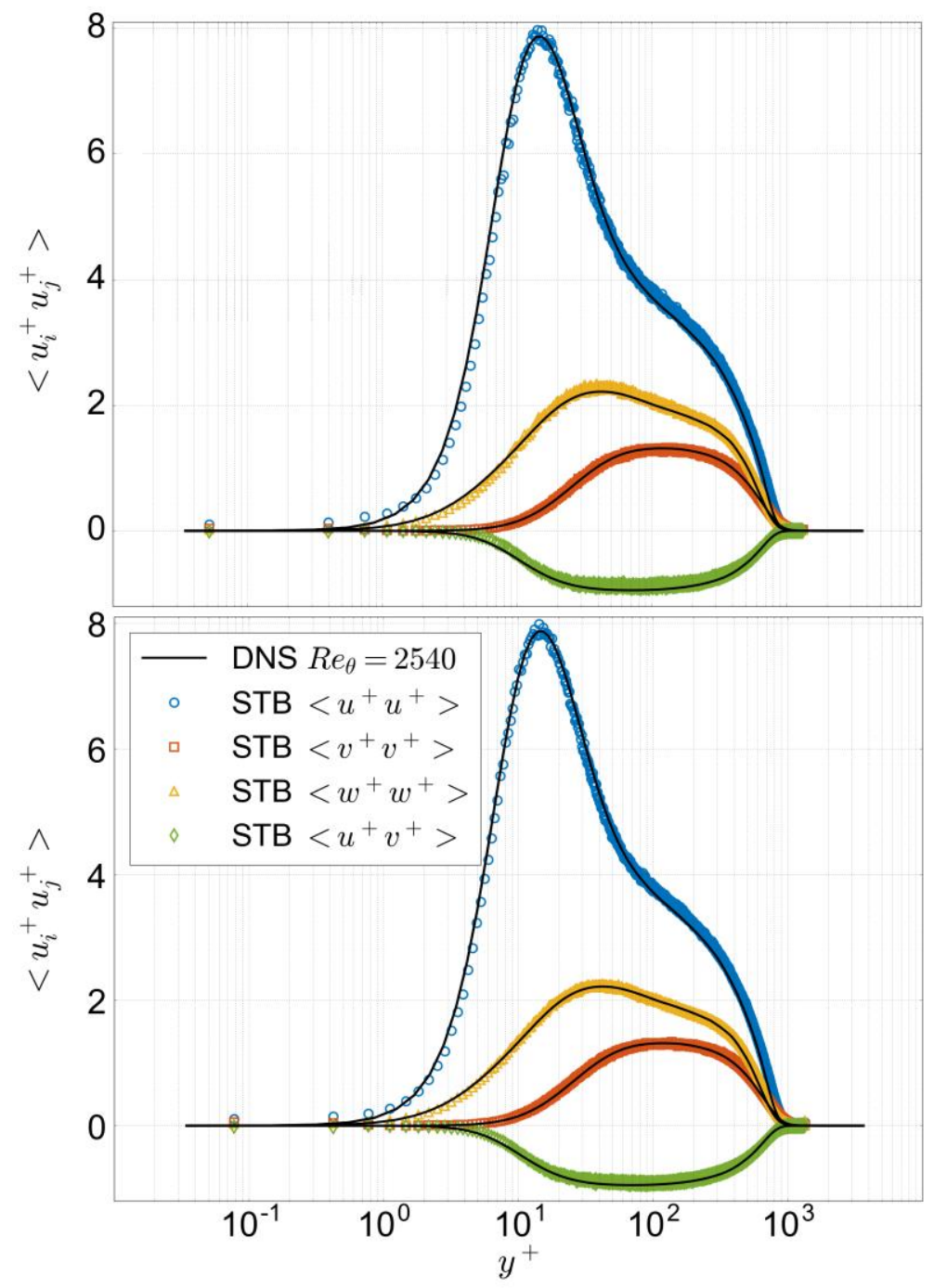

The 10,000 instantaneous track fields are analyzed by means of an ensemble averaging approach; due to the low acquisition frequency $(10 \mathrm{~Hz})$, the results from different recordings can be considered statistically independent. For each individual track, the particle locations along the four-pulse sequence are fitted by means of a second order polynomial and the position and velocity information are extracted at the mid-point location of the fitted track. The 3D domain is partitioned in one-dimensional bins of $14.5 \mu \mathrm{m}$ size along the wall-normal direction $(Y)$; the averaging is performed over all the individual samples found within the spatial region defined by each bin. An average number of approximately 35,000 individual samples from the 10,000 independent recordings is found in each bin. Given the chosen resolution, approximately three measurement points are located within a single wall unit $\left(y^{+} \approx 42 \mu \mathrm{m}\right)$.

The profiles relative to the turbulent fluctuations are shown for both pulse-separation strategies in Figure 5, together with the DNS results from Schlatter and Örlü (2010) at a very similar Reynolds number. Results show very good agreement with the DNS data; as expected a slightly higher noise level is found in the near wall region $\left(y^{+}<1.5\right)$, where the lower flow velocity leads to smaller particle displacements and, in turn, to a reduced dynamic range of 
the measurement. The mean deviation from the DNS solution along the profiles ranges between $1 \%$ and $2 \%$ of the peak value, depending on which component of the Reynolds stresses is considered; nevertheless, given the periodic boundary conditions, smooth wall and slightly different Reynolds number of the numerical simulation, the DNS solution cannot be used for a rigorous determination of the accuracy of the MP-STB results.

The lower resolution of the first two images when the system is operated in timing mode (Figure 5-bottom) does not seem to affect the accuracy of the results; in this respect, the choice of rotating the sensor in order to have the higher resolution aligned with the wall-normal direction, where velocity gradients are higher, plays a beneficial role.

The main difference between the two pulse-separation approaches lies in the number of particles that can be successfully tracked; when the polarization-based strategy is adopted, only $57 \%$ of the actual tracks are reconstructed by the Multi-Pulse STB. On the other hand, when the timing-based strategy is chosen, the percentage of detected tracks rises to approximately $70 \%$. The approximation is based on a comparison of the number of reconstructed four-pulse tracks in the measurement volume with the number of detected particle image peaks in the respective individual camera images. These figures are relative to the number of four-pulse tracks only; in both cases shorter tracks resulting from particles leaving or entering the investigated domain are discarded.

In the case of the timing-based approach roughly $30 \%$ of the expected number of particles are missing. The different camera sensitivity between the two systems (CCD sensor for the FOX cameras and sCMOS sensor for the PCO system) and the different viewing directions with respect to the Mie scattering lobes could lead to significant variations in the image particle peak intensity along the four recordings. As a consequence, a particle might be visible for one system (i.e. produce a particle image having a peak intensity above the chosen intensity threshold), but not for the other, therefore failing to be reconstructed. These effects appear to be amplified by the use of polarization filters in front of the camera lenses, accounting for the loss of an additional $13 \%$ of tracks. Besides reducing the spatial resolution of the measurement, the loss of tracks directly affects the iterative processing strategy of the Multi-Pulse STB (Novara et al 2016b); in fact, particle images which do not correspond to complete fourpulse tracks remain in the residual images and do not contribute to reducing the particle image density for the next STB iterations. When dealing with higher particle image densities, this problem could lead to an overall reduction of the accuracy in determining the particle position by means of IPR and affect the quality of the velocity and acceleration measurements.

These reasons motivated the implementation of the multi-exposed recording strategy, where the use of a single 3D imaging system guarantees comparable reconstruction performances for all pulses therefore increasing the consistency of the particle peak intensity along the tracks. The performances of this latter method are assessed experimentally by analyzing double-exposed recordings from a turbulent boundary layer investigation at $15 \mathrm{~m} / \mathrm{s}$; results are presented and discussed in the following section. 
Figure 6. Experimental setup at the SWG facility (DLR Göttingen). Top: illuminated volume and flow direction. Bottom: eight-camera imaging system placed below the wind tunnel; cameras indicated by blue circles (dashed line for those not visible in the picture) and flow direction indicated by the yellow arrow.
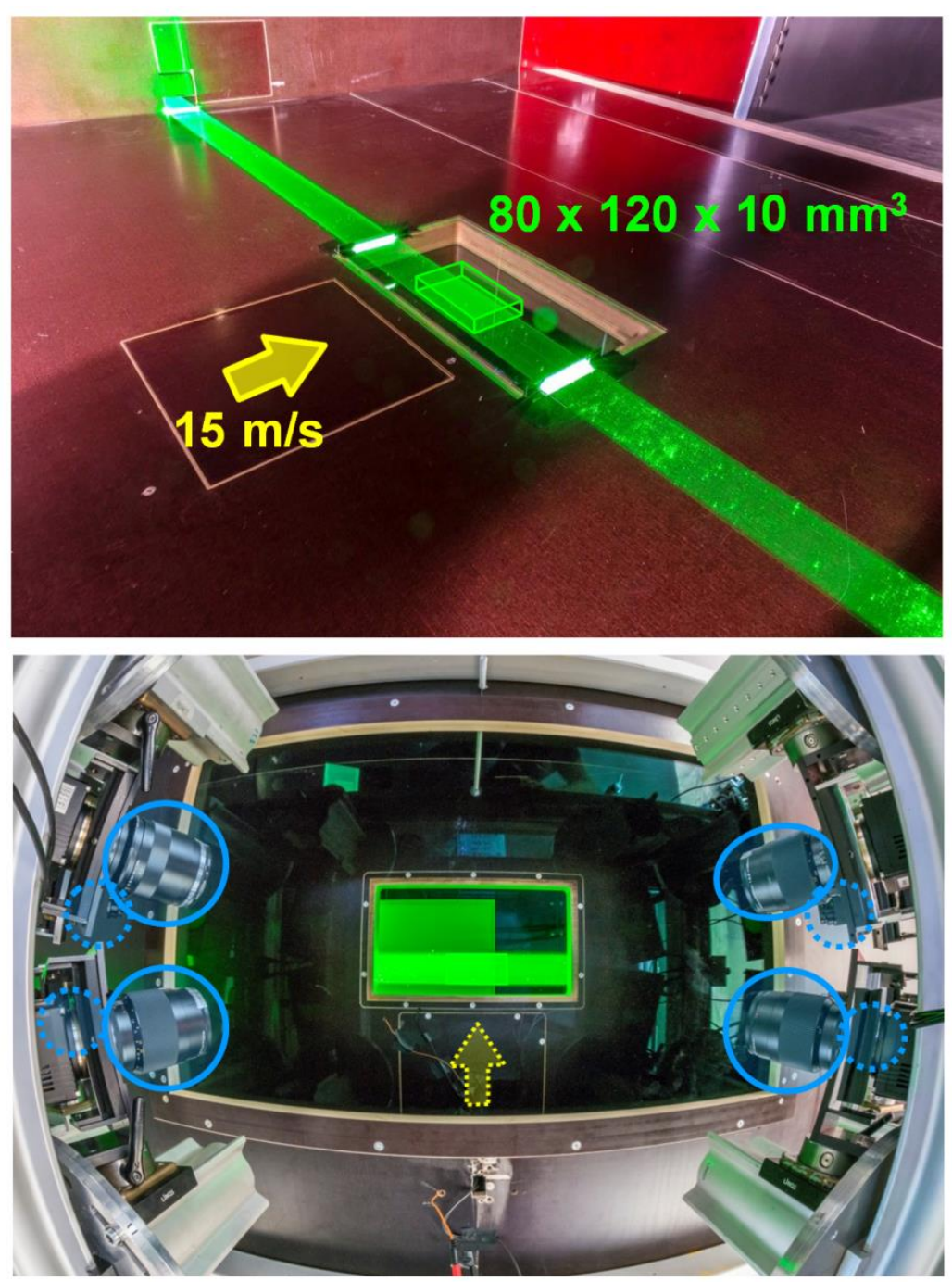

\section{Experimental assessment of the multi-exposed frames acquisition strategy}

The timing diagram for the multi-exposed acquisition strategy is shown in Figure 2c; a single imaging system is employed and each frame is double-exposed to capture the four pulses. The iterative strategy for reconstruction and tracking shown in Figure 1 is applied and adapted to the case of multi-exposed frames; the feasibility of the application of the MP-STB processing technique to such data is demonstrated by analyzing multi-exposed particle images from a turbulent boundary layer investigation at $15 \mathrm{~m} / \mathrm{s}$ carried out in the Cross-Wind Test Facility (SWG) at DLR Göttingen. A single imaging system consisting of 8 PCO Edge sCMOS cameras $(2560 \times 2160 p x, 6.5 \mu \mathrm{m}$ pixel size) is used to image a $80 \times 120 \times 10 \mathrm{~mm}^{3}$ volume in stream-wise $(X)$, span-wise $(Y)$ and wall-normal $(Z)$ directions, respectively (Figure 6). The flow is seeded with DEHS particles and the digital resolution is approximately $23 \mathrm{px} / \mathrm{mm}$. An uneven pulse distribution is chosen where pulses $1-2$ and $3-4$ are separated by $50 \mu s$ while the time separation between pulses 2 and 3 is $150 \mu s$. 
Figure 7. Detail of double-exposed recording (full frame size is $2560 \times$ $2160 p x)$ for $0.024 p p p$.

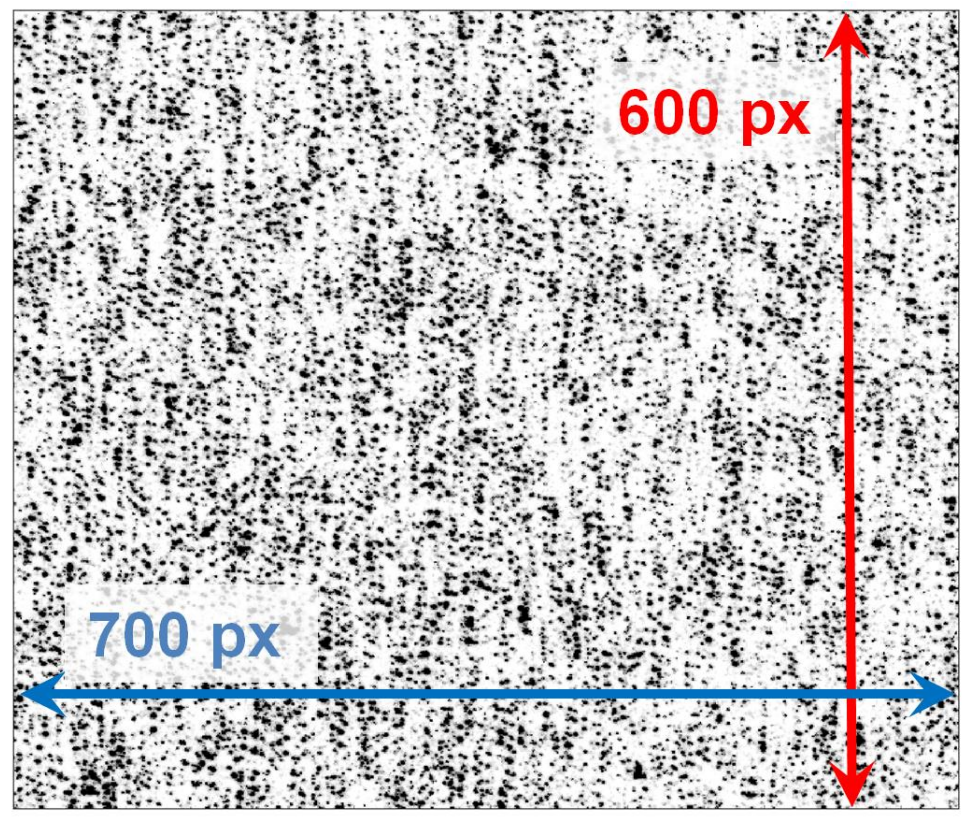

Two particle image density levels are applied; $0.024 p p p$ and $0.046 p p p$. The low seeding concentration images are used to accurately estimate the percentage of reconstructed tracks by MP-STB while the more seeded ones provide higher spatial resolution for the visualization of instantaneous $3 \mathrm{D}$ flow structures. These numbers refer to the particle image density of the double-exposed recordings; when the particle image density values are used to estimate the number of tracks expected within the investigated volume, the fact that each tracer results in two particle images on the double-exposed frames needs to be taken into account.A detail of a recorded double-exposed image is presented in Figure 7 for the low particle image density case. Given the size of the active portion of the sensor (i.e. illuminated region common to all cameras, approximately $2440 \times 1810 p x$ ) around 53,000 actual particles are expected within the investigated domain.

The particle tracking approach described in Novara et al (2016a, 2016b) is adapted to the case of multi-exposed recordings as each IPR object (for the first and second frame respectively) contains two realizations of the same particle distribution at two subsequent time instants. The adapted approach for particle tracking is depicted in Figure 8 and Figure 9 for the nearest neighbor approach and for the case where a predictor of the velocity field is available, respectively. Reconstructed particles for pulses $1-2$ and $3-4$ are shown by black and gray solid dots, respectively, search radii for two- and four-pulse tracks $\left(\delta_{2 p}\right.$ and $\left.\delta_{4 p}\right)$ are indicated by black dashed lines. The blue arrows indicate two-pulse track candidates while the dashed blue lines represent the linear extrapolation of the twopulse candidates towards the central time of the four-pulse sequence (the hollow markers show the extrapolated location, where the four-pulse tracks search area is centered). The orange line represents the polynomial fit (typically second order) of the particle locations along the complete four-pulse track. The yellow arrows in Figure 9 indicate the predicted displacement of the particles obtained from a velocity predictor, when available. 


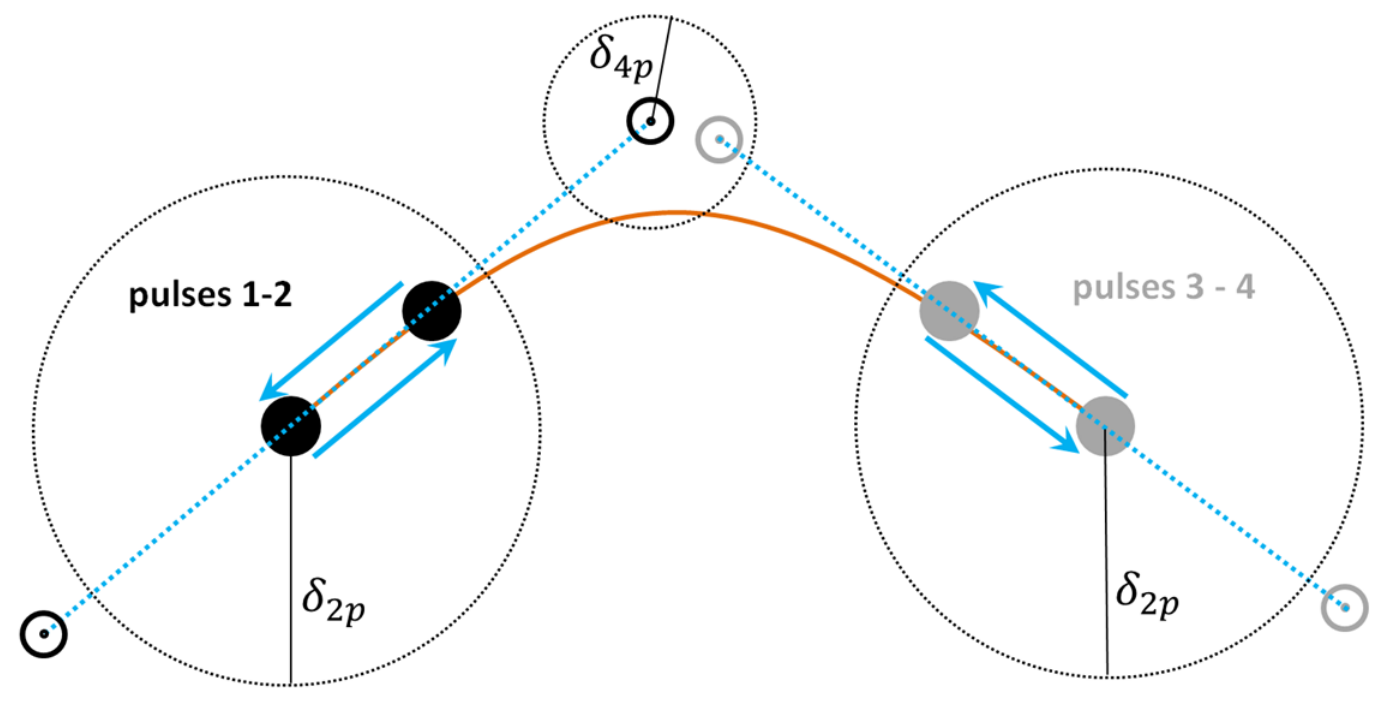

Figure 8. Particle tracking scheme for multi-exposed recordings performed without the aid of a velocity predictor.

The tracking step is divided into two phases: the definition of two-pulse track candidates and the identification of four-pulse tracks. If a predictor for the particle displacement is not available (Figure 8), the two-pulse candidate search is carried out establishing a search radius around each reconstructed particle $\left(\delta_{2 p}\right)$.

Due to the double-exposed particle images, no knowledge of the particle direction is available; as a consequence, for each couple of particles within the same IPR object, two possible two-pulse track candidates are (potentially) created. The ambiguity for the flow direction is solved when two-pulse track candidates are combined into fourpulse tracks. Each two-pulse candidate is linearly extrapolated to the mid-time-point of the sequence, where, assuming that the first pulse is recorded at time $t=0 \mu \mathrm{s}$ :

$$
t_{\text {mid-point }}=\frac{d t_{12}+d t_{23}+d t_{34}}{2}=125 \mu \mathrm{s}
$$

Forward extrapolation is applied for pulses $1-2$ and backward for pulses $3-4$; only the candidates corresponding to the actual particle direction will produce mid-point locations falling both within the chosen search region $\left(\delta_{4 p}\right)$.

Once the coupled two-pulse candidates are matched, the location of the four particles along the track is fitted by means of a second order polynomial fit; in case multiple four-pulse track candidates are found which share one or more particle (a situation commonly encountered when adopting large search radii), the candidate showing the lowest residual from a linear fit of the particles location along the sequence is chosen (following the approach proposed by Schanz et al 2016). The use of the mid-point prediction to combine the two-pulse candidates is justified by the choice of an uneven pulse separation, where pulses 2 and 3 are separated by a time interval typically 3 to 4 times larger than pulses $1-2$ and 3-4. In case a predictor for the particle displacement is available (e.g. a constant shift, velocity field from Particle Space Correlation - PSC, Novara et al (2016b) - or from four-pulse tracks from a previous STB iteration), the search radius for the two-pulse candidates' definition can be reduced and the chance of ambiguous particle direction decreases, Figure 9. 


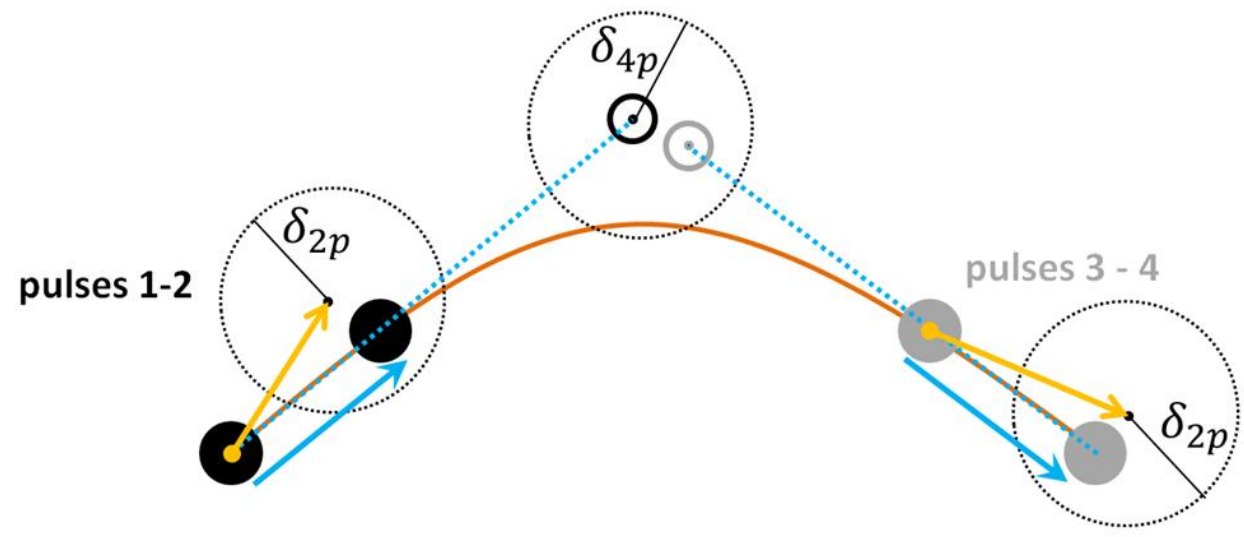

Figure 9. Particle tracking scheme for multi-exposed recordings aided by a velocity predictor from Particle Space Correlation (yellow arrows).

Four MP-STB iterations are applied here to analyze both the low and high particle image density recordings; images are preprocessed by means of minimum image subtraction. For each STB iteration, five IPR iterations are performed to triangulate the position of particles whose image peak value is larger than 70 counts; the allowed triangulation error was set to $1.2 p x$. The settings for the particle tracking parameters of MP-STB are indicated in Table 1 together with the approximate number of tracks reconstructed after each STB iteration for the low particle image density case. After the first IPR reconstruction of the two frames, a velocity vector field is obtained by means of Particle Space Correlation (Novara et al 2016b). A 3D cross-correlation with multi-grid window deformation (Scarano and Riethmuller 2000) is performed between the two particle objects; unlike for tomographic PIV, where the cross-correlation is applied to the voxel space, the PSC makes use only of the particle peak location and intensity as obtained from the IPR. Large cross-correlation windows are chosen in order to cope with the large particle displacement within the $200 \mu s$ time separation (up to $60 p x$ ); the final cross-correlation window size is approximately $5 \times 5 \times 1 \mathrm{~mm}^{3}(120 \times 120 \times 30 \mathrm{px})$ in the stream-wise, span-wise and wall-normal directions respectively.

The velocity field from PSC is used as a predictor for the particle displacement in the first STB iteration; a tri-linear interpolation is used to evaluate the predictor field at the location of the reconstructed particles. Approximately $80 \%$ of the total number of expected tracks is found at the first MP-STB iteration. These tracks are used as a predictor for

Table 1 Parameters setting and number of reconstructed tracks for the iterative MP- STB processing $(0.024 p p p)$.

\begin{tabular}{l|lccc}
\hline MP-STB iteration & Predictor & $\delta_{2 p}[p x]$ & $\delta_{4 p}[p x]$ & Number of tracks $(\sim)$ \\
\hline 1 & PSC field & 4 & 5 & 43,000 \\
2 & tracks from iter 1 & 6 & 6 & 3,600 \\
3 & no predictor & 15 & 6 & 2,000 \\
4 (two-pulse tracks) & no predictor & 8 & - & 400
\end{tabular}


the following iteration, delivering additional $3,600(\approx 6.7 \%)$ tracks.

As the initial prediction was obtained by means of a low-resolution cross-correlation approach (PSC), particles within regions characterized by strong velocity gradients (e.g. near-wall region) are not tracked in the first two iterations. As a consequence, the third iteration is performed without the aid of a predictor, but following a nearest neighbor approach (Figure 8) with a larger search radius for the two-pulse track candidates' identification. Most of the $2,000(\approx 3.7 \%)$ tracks identified during the third iteration are low-speed tracks found in the vicinity of the wall. When the flow velocity results in particle displacements lower than a single particle image diameter $(\approx 2 p x)$ between the closer pulses $(1-2$ and $3-4)$, the particle images of the same tracer on the double-exposed frame start overlapping eventually leading to a single particle image. As a consequence, two-pulse tracks cannot be identified within the double-exposed IPR object. In order to track these particles and recover the information about low velocity flow regions, the last STB iteration is performed identifying two-pulse tracks between the particles reconstructed at pulses $1-2$ and the ones at $3-4$. A maximum displacement of approximately $8 p x$ is expected within the time separation between $1-2$ and $3-4(200 \mu s)$ for particles experiencing a maximum displacement of $2 p x$ over the shortest time separation of $50 \mu s$. Therefore, a search radius of $8 p x$ is established for the two-pulse track search and approximately $400(\approx 0.7 \%)$ two-pulse tracks are found, as expected, in the near-wall region. A linear fit is used to estimate the flow velocity from the two-pulse tracks and no information about the particles acceleration can be extracted. This final iteration accounts for $10 \%$ of the total computation time of the STB processing of a single four-pulse recording.

The image matching scheme based on the minimization of the particle residual images adopted during IPR to correct the triangulated particle locations (shaking step) makes use of the Optical Transfer Function (OTF, Schanz et al 2013) estimated during Volume Self Calibration (VSC, Wieneke 2008). Unlike as for single-exposed frames where overlapping situations might occur only for one particular viewing direction, overlapped particle images resulting from slow flow velocity on multi-exposed frames will appear simultaneously on all camera recordings of the imaging system. Adopting single particle OTFs during the minimization of the residual during IPR could affect the accuracy of the particle peak positioning. The use of an adapted OTF for particle image doublets can be envisioned, where the overlapping particle images are not treated as single tracers, but the shaking step is carried out for the two particles simultaneously, and the total residual of the particle image couple is minimized. An iterative procedure can be implemented, where an initial estimate of the displacement from single particle OTF reconstruction is used to produce an overlapped OTF image obtained superimposing two single particle OTFs separated by the given displacement estimate. The correction of the particle image doublets peak location based on the overlapped OTF will lead to a new estimate of the particle displacement over the four-pulse tracks which can be used for the subsequent iteration. The possible beneficial effect of the adaptive overlapped-image-OTF should be weighed against the resulting increase in computational time caused by its iterative nature. The investigation of this novel OTF approach by means of the analysis of synthetic recordings goes beyond the scope of the present study. 

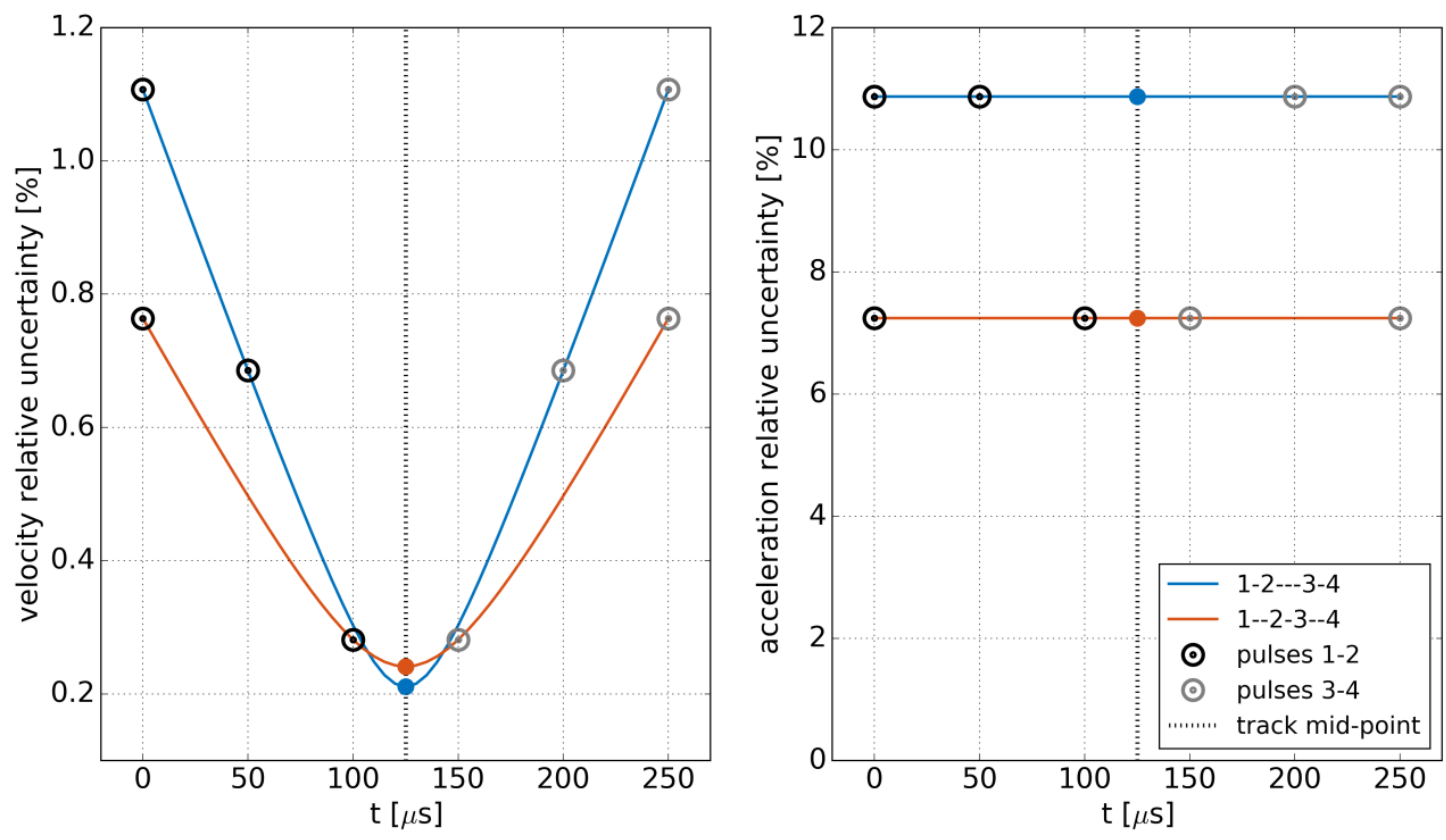

Figure 10. Velocity (left) and acceleration (right) relative uncertainties for two different pulses distributions along the same time span of $250 \mu \mathrm{s}$; markers indicate the location of the pulses along the four-pulse sequence and the effective measurement point at the center of the tracks. A single dash symbol in the legend labels indicates a $50 \mu$ s time separation (e.g. $1-2---3-4$ ).

Alternatively, a different timing strategy where the separation between pulses $1-2$ and $3-4$ is increased and pulses 2 and 3 are separated by a smaller $d t$ can be envisioned (communication with Bernd Wieneke) to avoid resorting to less accurate two pulse tracks for the low-velocity regions. In this situation, the tracking scheme would involve at first the identification of the two-pulse tracks between the second and third pulse, followed by the extension of the tracks outwards to complete the four-pulse tracks reconstruction by means of linear extrapolation and the definition of suitable search radii.

An assessment of this alternative tracking strategy is necessary before any conclusion can be drawn regarding its performances, mainly in terms of track yield, and it is devoted to a future study. On the other hand, some considerations regarding the distribution of the pulses over the observation time can be made based on the uncertainty analysis of the velocity and acceleration information extracted from the four-pulse tracks fit. The positional error of the reconstructed particles can be estimated by means of synthetic experiments; based on the results presented by Novara et al (2016b), where realistic imaging conditions were mimicked (e.g. image noise), the particle peak position error is approximately $0.15 p x$. The fitting of the particle positions along the track, the derivation and the evaluation of the polynomial fit at an arbitrary point in time along the track can be combined into a single step, and expressed as a weighted sum of the measured locations, where the weights are specific to the point in time and to the derivation order. Assuming that the positional error is not correlated between different time steps, and that a second order polynomial fit is suitable to describe the particle dynamics, the initial estimate for the 
positional error can be used, together with the weights, to derive the velocity and acceleration uncertainties by means of Gaussian uncertainty propagation.

The results of the uncertainty propagation analysis are presented in Figure 10, where the velocity and acceleration uncertainty have been evaluated at arbitrary points along the time-span of the four-pulse sequence (i.e. $250 \mu s$ from pulse 1 to pulse 4). The relative uncertainties have been computed with respect to a reference velocity (free stream, $15 \mathrm{~m} / \mathrm{s}$ ) and a reference acceleration of $12,000 \mathrm{~m} / \mathrm{s}^{2}$, which has been obtained based on the histogram of the acceleration magnitude; in particular, in order to exclude possible outliers, it has been chosen as the $99.75^{\text {th }}$ percentile. As expected, for both pulses distributions $(1-2---3-4$ and $1--2-3--4$, where the dash symbol indicates one $\Delta t$ of, in this case, $50 \mu s$ ) the highest accuracy for the velocity measurement is attained at the center of the track and comparable for both distributions. Given the second order polynomial fit, the acceleration error is constant; however it can be observed that when a shorter separation is adopted between pulses 2 and 3 , a significant reduction of the acceleration uncertainty $(\approx 35 \%)$ can be attained, which, together with the lower chance of generating overlapping particle images within the double-exposed frames, suggests that the $1--2-3--4$ pulse separation strategy could be a suitable choice for the recording of multi-exposed images for MP-STB. On the other hand, for the results presented in the remainder of the present study, the reference values are those from the $1-2---3-4$ strategy (blue curve in Figure 10).

At the end of the MP-STB iterative strategy, approximately 49,000 instantaneous tracks are identified, corresponding to $92 \%$ of the expected number of particles estimated from the recorded images. These results are confirmed by the similar performances of the reconstruction process when dealing with higher particle image densities. In fact, when the same processing parameters are applied to the $0.046 p p p$ case, roughly 95,000 instantaneous tracks are successfully reconstructed; the increase in the number of retrieved tracks is consistent with the increase in particle image density.

Due to the many differences in the experimental setup between the present investigation and the applications presented in the previous section, a direct comparison in terms of percentage of retrieved tracks cannot be carried out as these figures are affected by several experimental and processing parameters (e.g. number of cameras, sensor type, viewing directions, illumination strategy and particle image density). Nevertheless, the results from the multiexposed strategy seem to indicate that, despite the more challenging task of tracking particles within double-exposed recordings, the use of a single imaging system is less prone to suffering from loss-of-tracks than double-imaging system approaches.

An instantaneous result from MP-STB is presented in Figure 11, where individual tracks are color-coded by the stream-wise velocity component; the presence of low- and high-speed streaks can be observed along the span-wise direction ( $Y$ axis), while low velocity tracks are found, as expected, in the near-wall region (Figure 11-bottom).

The scattered results extracted from the track fit at the mid-point of the individual particle tracks are used as the input for the FlowFit data assimilation algorithm (Gesemann et al 2016) in order to interpolate the result onto a regular grid and enable the visualization of the instantaneous 3D structures. The FlowFit algorithm makes use of a system of uniform, cubic B-splines for representing velocity and pressure fields and leverages physical constraints derived from the Navier-Stokes equations to increase the spatial resolution of the interpolation beyond the sampling 

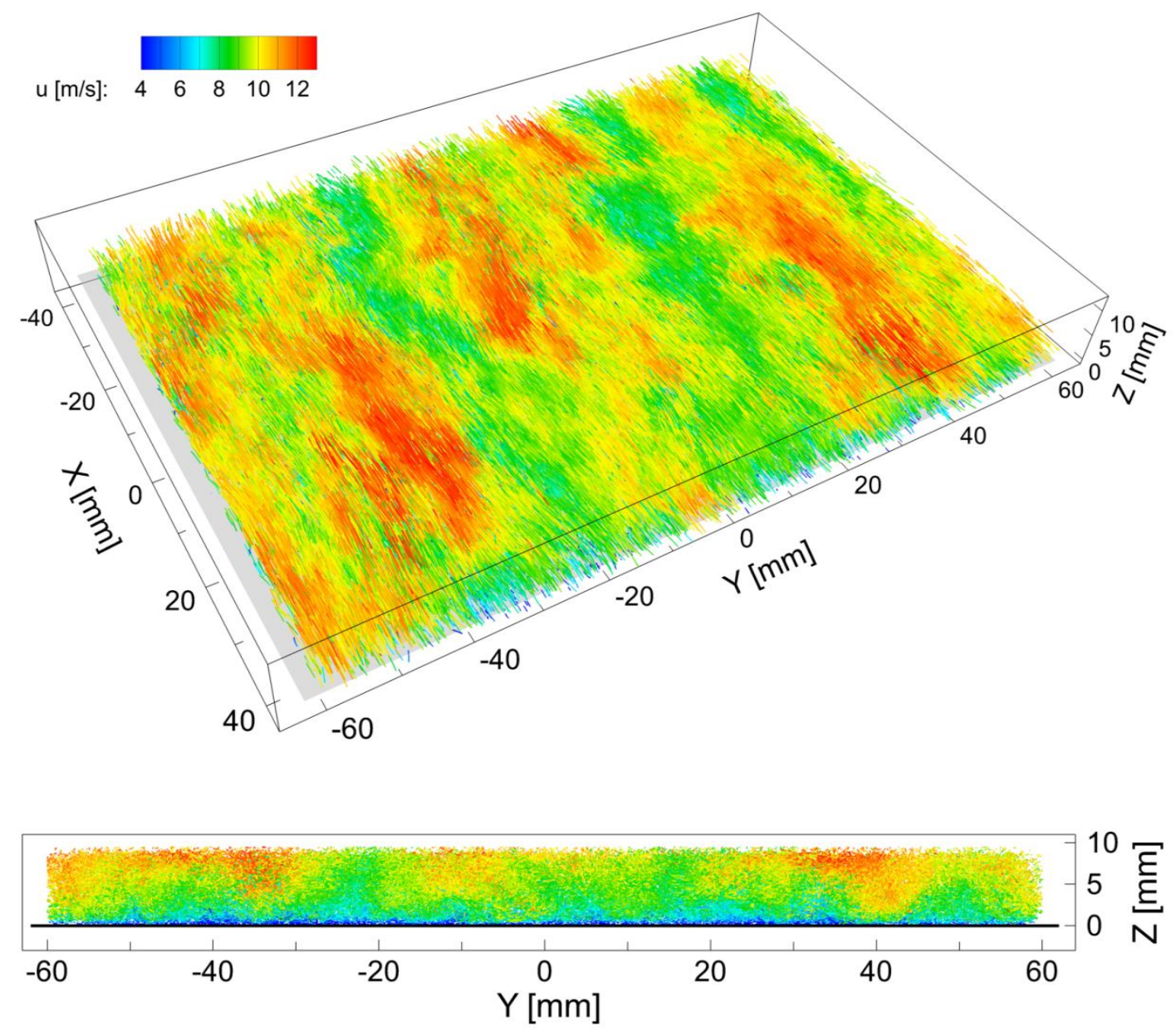

Figure 11. Two views of an instantaneous result from MP-STB at $0.046 p p p$ (multi-exposed recordings); approximately 95,000 tracks are shown color-coded with the stream-wise velocity component (along the Xaxis). Wall surface is indicated by the gray plane (top) and black line (bottom).

offered by the scattered tracked particles (each B-spline cell contains on average 0.1 tracked particles); spatial gradients can be analytically evaluated from the B-spline functions.

The instantaneous 3D pressure distribution can be recovered as part of the FlowFit data assimilation and interpolation; several reconstruction strategies, differing in complexity of the involved data and in the applied regularizations, are possible and described in detail in Gesemann et al (2016) and Huhn et al (2017). In the present study, pressure has been reconstructed by the FlowFit method by jointly optimizing velocity and pressure field (including boundary conditions) based on measured particle velocities and accelerations. With the help of the Navier-Stokes momentum equation, acceleration can be expressed in terms of the pressure gradient and the viscous term. This allows incorporating the measured particle acceleration data as input to FlowFit to further increase the spatial resolution but also disambiguate the pressure field on the boundary of the volume. Finally, an offset correction only (e.g. based on known flow conditions in the measurement volume) needs to be applied for a correct pressure estimation. 


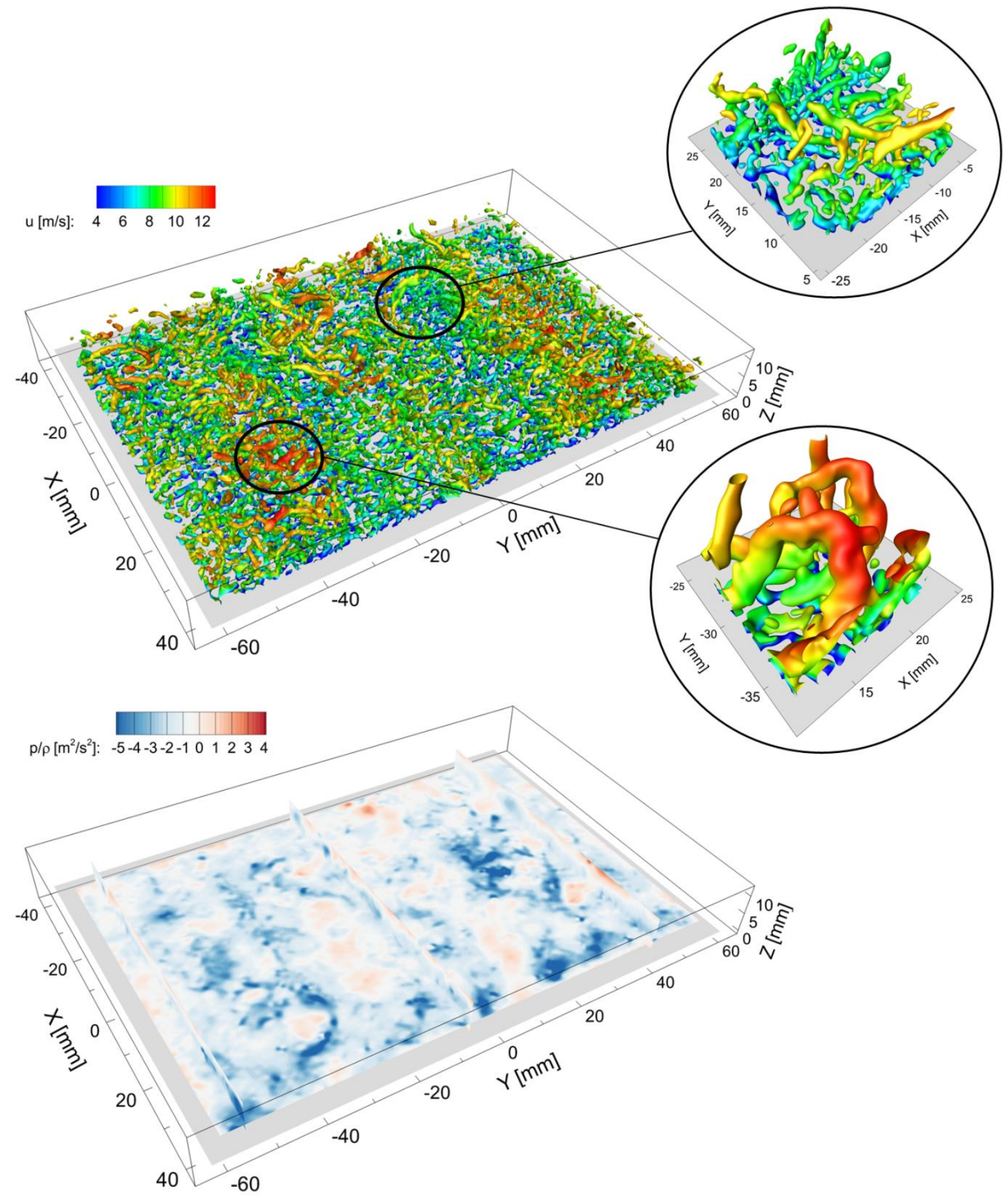

Figure 12. FlowFit interpolation onto regular grid. Top: instantaneous flow structures visualized by isosurface of Q-criterion $\left(1,200,0001 / s^{2}\right)$ colored by the stream-wise velocity component. Details of flow structures viewed from a different perspective visualized in the zoomed region indicated by black circles. Bottom: contours of pressure normalized by density $\bar{p}$ over wall-parallel (at $Z=2.5 \mathrm{~mm}$ ) and wall-normal slices (at $Y=-53 /-2.5 / 48 \mathrm{~mm}$ ). 
An instantaneous result from FlowFit is shown in Figure 12; the algorithm is applied to the high particle image density case result from MP-STB presented in Figure 11. An iso-surface of $Q$ criterion is plotted, color-coded by the stream-wise velocity component (Figure 12-top). The level of detail that can be achieved in the evaluation of the spatial gradients from the FlowFit regularization of the relatively sparse tracking data can be appreciated in the zoomed-in regions shown in Figure 12-top, the bottom one resembling, in term of shape and inclination, the horseshoe-shaped vortical structures described in literature as Hairpin vortexes (Adrian 2007 among others).

Instantaneous contours of $\bar{p}$ (pressure normalized by density) on two-dimensional wall-parallel and wall-normal planes are presented in Figure 12-bottom; the low pressure regions associated with the vortical structures in the boundary layer can be identified as well as the higher pressure zones corresponding to the low velocity streaks.

From the instantaneous results presented here, it can be concluded that the acquisition strategy based on multiexposed recordings, combined with the MP-STB algorithm, allows for the identification of the majority of expected particle tracks. This is due to the higher consistency in terms of particle peak intensity along the recording sequence offered by the use of circular polarized laser light and a single imaging system, which reduces the chances of lossof-tracks experienced when using the dual imaging system approaches described in the previous section.

Despite the limitations to the seeding concentration applicable for multi-exposed investigations imposed by the artificial increase in the particle image density on double exposed recordings, data assimilation techniques (as FlowFit -Gesemann et al 2016 - or VIC+ - Schneiders and Scarano 2016) can be applied to the scattered tracking results in order to accurately reconstruct the instantaneous flow structures on a regular grid.

In order to provide a further reference for MP-STB measurements with multi-exposed recordings, the investigation of a turbulent boundary layer flow with adverse pressure gradient is presented in the following section, where results in terms of boundary layer statistics from MP-STB can be directly compared to planar PIV results obtained in the same facility under the same experimental conditions.

\section{Application of MP-STB for multi-exposed recordings in a turbulent boundary layer with adverse pressure gradient}

The experiment was carried out at the Eiffel type atmospheric wind tunnel (AWM) of the University of Armed Forces (Munich). The objective of the measurement is to provide accurate experimental data of adverse pressure gradient turbulent boundary layer (APG-TBL) at high Reynolds numbers $\left(10,000<R e_{\vartheta}<40,000\right)$ that can be exploited for validation of numerical simulation models. Several experimental techniques were implemented in the framework of the same measurement campaign (referred to as VICTORIA), namely planar PIV, time-resolved 2D and 3D-STB and Multi-Pulse STB. When the spatial resolution of the measurement is considered, 2D-PIV is only suitable in regions with relatively low velocity gradients (e.g. outer flow region of the TBL) while highmagnification STB can deliver accurate Lagrangian particle tracking data in regions characterized by strong gradients (e.g. near-wall) over a reduced field-of-view (FOV). The MP-STB technique was introduced to fill the gap between the two approaches and deliver instantaneous 3D particle tracks over a relatively large volume both encompassing the whole TBL development along the wall-normal direction and providing insight into the 


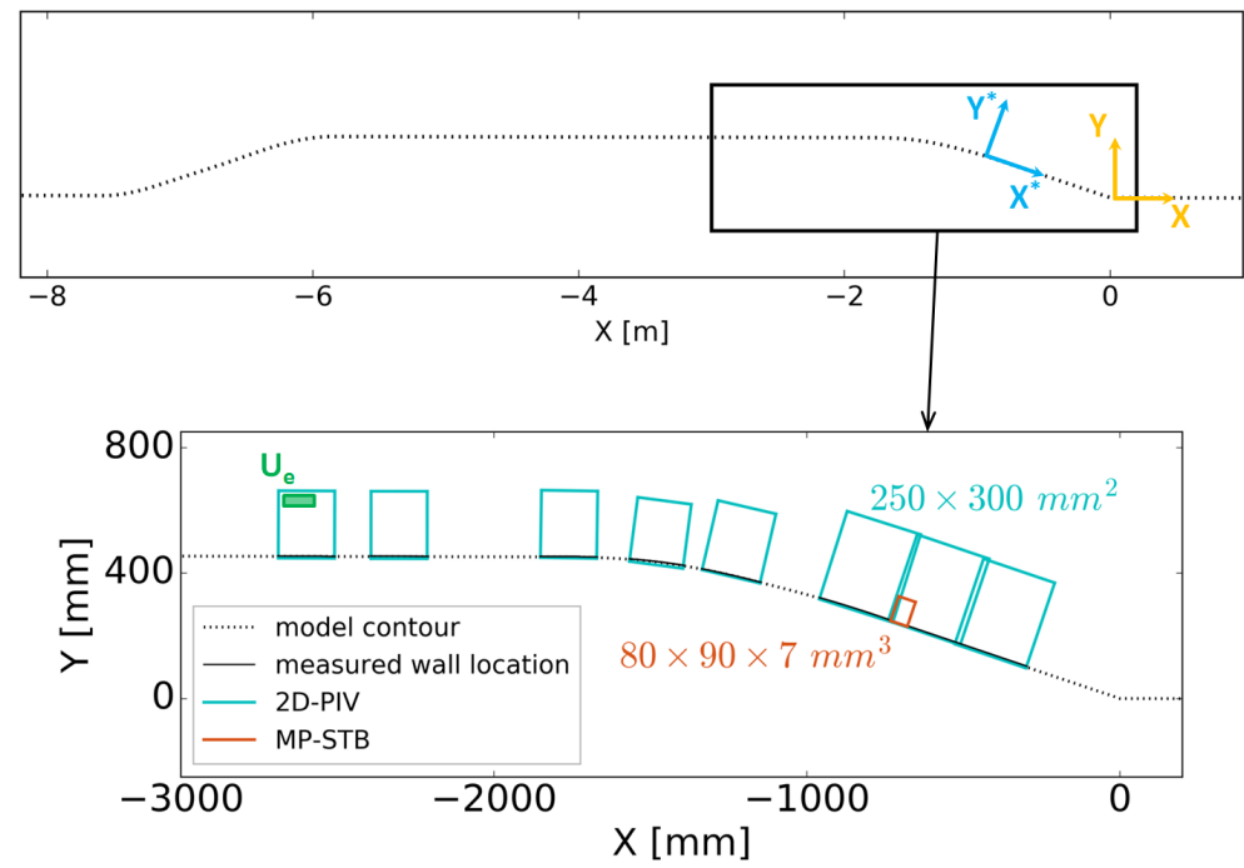

Figure 13. Top: contour of the wall model for the TBL with adverse pressure gradient investigation; wind-tunnel $(X, Y)$ and wall $\left(X^{*}, Y^{*}\right)$ reference systems indicated in orange and blue respectively. Flow is aligned with $X$ axis. Bottom: fields-of-view of the planar PIV and MP-STB systems in the zero (ZPG), favorable (FPG) and adverse pressure gradient (APG).

development of the flow along the stream-wise (wall-parallel) direction. The present study focuses on the planar PIV and MP-STB results; a detailed description of the full measurement campaign can be found in Schröder et al (2018). A model, whose contour is shown in Figure 13-top, is installed on one of the vertical walls of the $1.8 \times 1.8 \mathrm{~m}^{2}$ test section; the model encompasses the entire span of the test section and approximately $8 \mathrm{~m}$ of the total test section length $(22 \mathrm{~m})$. After a zero-pressure-gradient (ZPG) region, the flow follows two slight curvilinear deflections over a length of $1.17 \mathrm{~m}$, which initially cause a small favorable-pressure-gradient (FPG), and enters into the adversepressure-gradient (APG) region over a subsequent flat plate with an inclination angle of approximately $18^{\circ}$. A global wind-tunnel reference system $(X, Y)$ is centered at the down-stream end of the model, where the contour joins the wind-tunnel wall; the $X$ axis is aligned with the flow direction within the zero pressure gradient (ZPG, $-5<$ $X<-2 \mathrm{~m}$ ). A local reference system can be defined for each point along the contour, where the $X^{*}$ and $Y^{*}$ are aligned with the wall-parallel and wall-normal directions, respectively.

Four boundary layer edge velocities have been investigated $\left(U_{e}=21.07 / 26.61 / 29.25 / 35.48 \mathrm{~m} / \mathrm{s}\right)$, where $U_{e}$ indicates the average wall-parallel velocity component measured within a region located in the top-left corner of the FOV of the most upstream PIV camera (green area in Figure 13-bottom). This value was monitored during all measurements (both PIV and STB) in order to ensure the stability of the wind-tunnel conditions and allow for a direct comparison of the results; the standard deviation of the control velocity $U_{e}$ during each run was found to be less than $0.08 \mathrm{~m} / \mathrm{s}$. In the present study, only results from the $U_{e}=29.25 \mathrm{~m} / \mathrm{s}$ case are presented. 


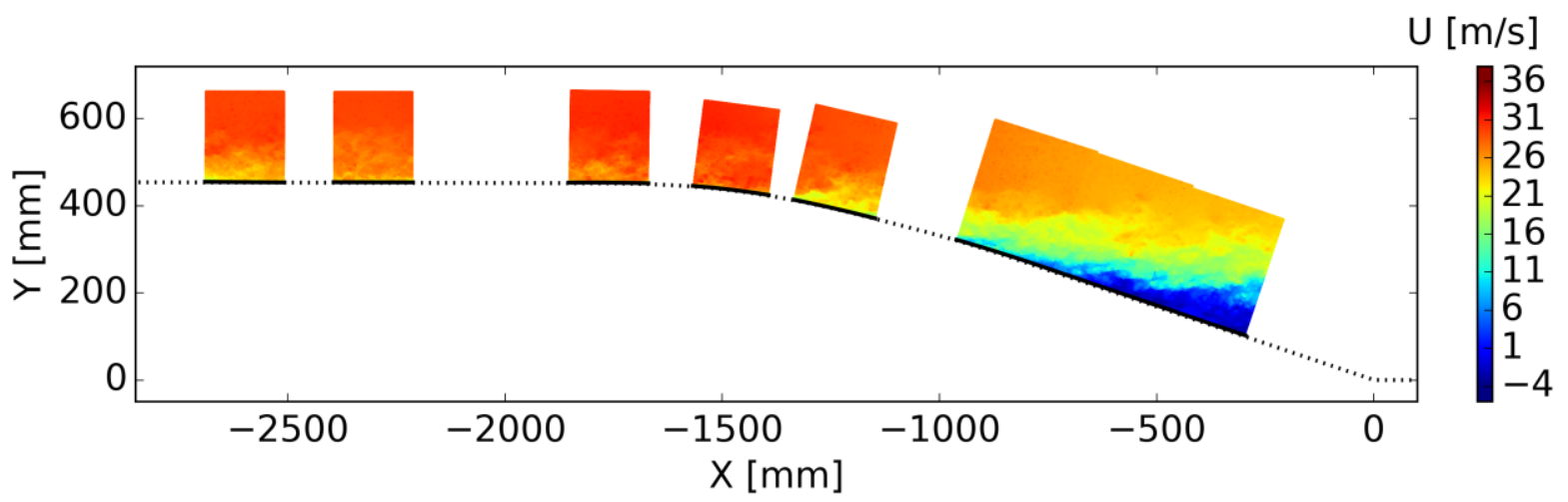

Figure 14. Instantaneous planar PIV result; contours of stream-wise $(X)$ velocity component.

The flow was seeded with DEHS particles having a mean diameter of $1 \mu \mathrm{m}$. Eight PCO Edge sCMOS cameras (5.6 $M p x$ resolution) were employed for the planar PIV overview measurement and installed on the roof of the wind-tunnel test section. The cameras were equipped with 100 and $80 \mathrm{~mm}$ Zeiss lenses. The FOVs of the eight PIV cameras are shown in Figure 13-bottom; the magnification ranges between 11.8 (ZPG and FPG cameras) and $8.8 \mathrm{px} / \mathrm{mm}$ (APG cameras) resulting in FOVs of approximately $180 \times 220 \mathrm{~mm}^{2}$ and $250 \times 300 \mathrm{~mm}^{2}$ respectively. Laser illumination was realized with two overlapping double-pulsed Nd:YAG Evergreen 200 lasers $(\approx 400 \mathrm{~mJ})$; the light sheet was introduced from an upstream location through the opposite wind tunnel wall allowing for a tangential illumination of the APG region.

The sensor of each camera was rotated in order to be approximately aligned to the local contour of the wall; the actual location of the model surface was detected on the camera images (which deviates from the nominal model geometry of approximately $1 \mathrm{~mm}$ along the wall-normal direction) and was used to correct the location of the PIV fields during the coordinate transformation operation from the sensor reference system to the wind-tunnel system. The detected location of the wall is indicated for each PIV field-of-view with a black solid line in Figure 13-bottom. Particle images were analyzed by means of an iterative multi-grid 2D cross-correlation approach with window deformation in PIVview3.0 (Pivtec, http://www.pivtec.com); the window size was progressively reduced from $128 \times 128 p x$ to $24 \times 14 p x$ in wall-parallel and wall-normal directions respectively. Given the chosen magnifications, the final window size was approximately $2 \times 1.2 \mathrm{~mm}^{2}$ and $2.7 \times 1.6 \mathrm{~mm}^{2}$ in the ZPG and APG respectively. A $66 \%$ overlap factor was applied in both spatial directions and universal outlier detection (Scarano and Westerweel 2005) was applied. An instantaneous PIV result is presented in Figure 14; a sequence of 16,000 instantaneous recordings was acquired in order to evaluate the flow statistics in terms of mean velocity and turbulent fluctuations components.

After the acquisition of the planar PIV images, the last four downstream cameras have been moved and rearranged into a single 3D imaging system to acquire four-pulse sequences suitable for 3D Lagrangian particle tracking by MP-STB. The multi-exposed recording strategy shown in Figure 2c was adopted. A $80 \times 90 \times 7 \mathrm{~mm}^{3}$ volume (along the wall-parallel, wall-normal and span-wise directions, respectively) was illuminated by two BigSky 400 lasers providing four pulses (200 $\mathrm{mJ}$ each) and imaged by the four cameras at $90^{\circ}$ scattering angle. The laser light 
was introduced almost perpendicular to the aluminum surface of the model. The measurement volume was located at the APG region, within the FOV of the second-to-last downstream PIV camera, Figure 13-bottom. The digital resolution was approximately $29 \mathrm{px} / \mathrm{mm}$. In order to increase the dynamic velocity range of the measurement, an uneven pulse separation strategy was adopted, where the separation between the second and third pulse was three times larger than the one between pulses $1-2$ and $3-4$. For the chosen time separation $\left(d t_{1-2}=d t_{3-4}=30 \mu s\right.$, $d t_{2-3}=90 \mu \mathrm{s}$ ), the maximum particle displacement within the shortest $d t$ was approximately $12 \div 15 \mathrm{px}$.

As the objective of the MP-STB measurement was to deliver accurate boundary layer profiles, the instantaneous particle image density of the double-exposed recordings was kept low at an average value of $0.01 \mathrm{ppp}$. Instantaneous track data from MP-STB can then be collected into bins following an ensemble average approach in order to produce high-spatial resolution statistics. For each flow velocity a sequence of 40,000 four-pulse recordings was acquired. The volume self-calibration approach proposed by Wieneke (2008) was applied for each recording in order to correct for mean de-calibration errors and to calibrate the OTF (Schanz et al 2013).

Unsteady camera vibrations occurring during wind-tunnel operation resulted in instantaneous de-calibration errors up to $0.7 p x$. An instantaneous correction of the camera calibration maps was applied based on the Global Shake technique (Jahn 2017). This method is based on the simultaneous correction of the 3D position of all particles triangulated by IPR; the image matching scheme employed for single particles within the IPR (shaking step) is adapted here in order to minimize the back-projected residual intensities of all particles simultaneously. This allows for the identification of a single shift within the image plane which corresponds to the displacement of the camera caused by unsteady structural vibrations. The detected shift is then applied to the calibration maps of each camera within the 3D imaging system to compensate for the de-calibration error. A comparison with the results obtained from single-image self-calibration (Michaelis et al 2011) was carried out over a number of instantaneous recordings and confirmed the capability of the Global Shake method to compensate for instantaneous de-calibration.

Due to the laser light impinging on the aluminum surface of the model, significant reflections were found on the camera images in the proximity of the wall. In order to avoid the introduction of spurious reconstructed intensity from the strong reflections at the wall, a masking of the images was carried out (in combination with minimum image subtraction), where the intensity of the pixels within the wall reflection region were artificially set to zero. Nevertheless, as the reflection region affected the first $4 \div 6 p x$ from the model surface, the masking of the images prevented obtaining measurement points within the first $0.2 \mathrm{~mm}$ from the wall.

Four MP-STB iterations were applied and the same processing parameters as shown in Table 1 employed; the average number of tracks reconstructed by MP-STB was approximately 19,000, including around 1,000 two-pulse tracks. An instantaneous MP-STB result is presented in Figure 15, where individual tracks are color-coded by the wall-parallel velocity component.

The average separation bubble location was identified from the planar PIV average field as the region exhibiting negative values of mean wall-parallel velocity components $\left(U^{*} \leq 0\right)$; for the present case, the average separation position was estimated at around $X=-500 \mathrm{~mm}$. As a consequence, instantaneous flow separation effects (backflow) are expected within the FOV of the MP-STB measurement. This can be observed when inspecting the instantaneous tracks and confirms the capability of the tracking system to solve the ambiguities in terms of flow 
Figure 15. Instantaneous MP-STB result in wall-parallel and wallnormal coordinate system; approximately 19,000 3D tracks color-coded by wall-parallel velocity component (frontal view in the $X^{*} Y^{*}$ plane). The origin of the $X^{*}$ axis corresponds to the center of the MP-STB field-ofview $(X=-700 \mathrm{~mm})$.

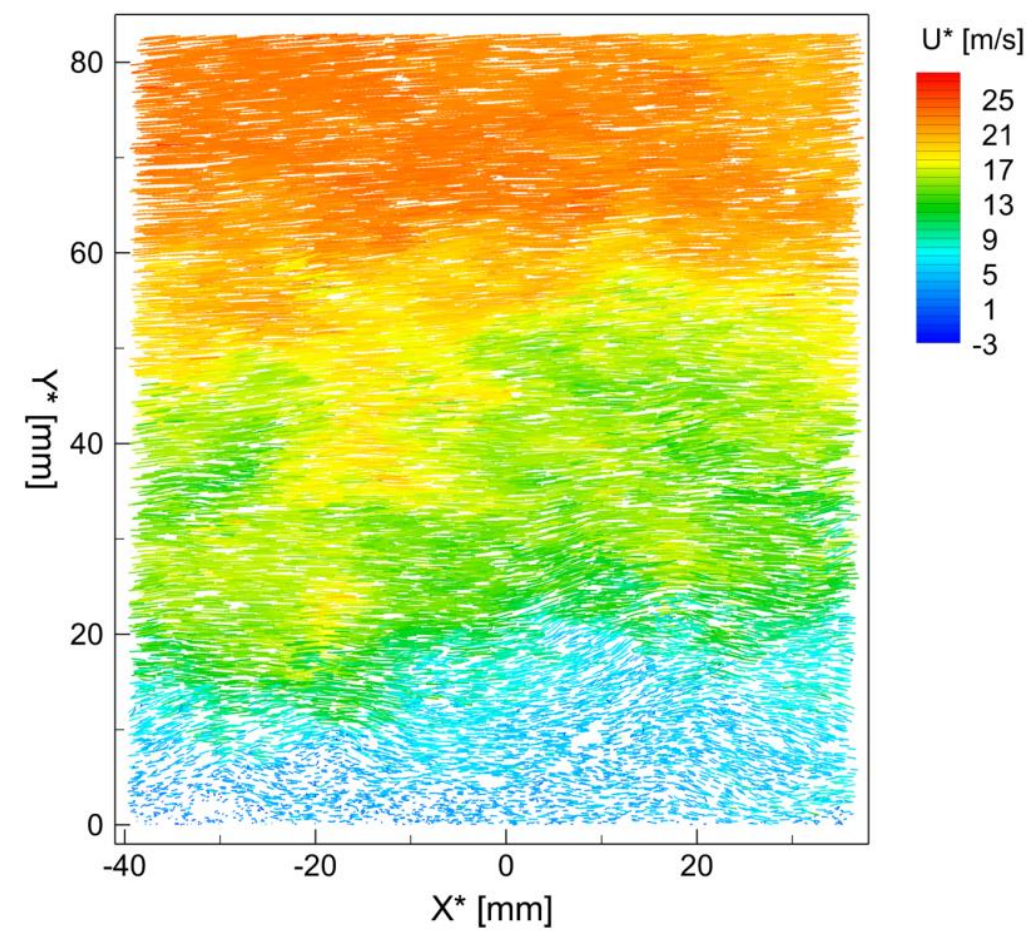

direction within the double-exposed frames by exploiting the complete four-pulse recording sequence. As a result of the intermittent flow separation, a significant impact of the two-pulse tracks $(\approx 5 \%$ of the total number of track, accounting for $30 \%$ of the total computational time) can be observed in the distribution of the velocity, particularly in the low velocity range (below $2.5 \mathrm{~m} / \mathrm{s}$ ), where, given the magnification and the time separation between the pulses, double-exposed particle images are expected to significantly overlap.

A relatively large unsteady displacement of the wall location (identified onto the camera images exploiting the wall reflections) was found along the recorded sequence (up to $6 p x$ ). As the instantaneous camera displacement, detected during the correction of the decalibration error, was typically below $1 p x$, a significant part of the wall image displacement can be ascribed to the vibration of the wind-tunnel walls during operation.

This artificial shift of the instantaneous track fields (mainly along the wall-normal direction) needed to be compensated for before the ensemble averaging could be performed along the 40,000 recordings sequence. Bright spots detected within the reflection region on the camera images were triangulated in the 3D space according to the corrected calibration maps; approximately 500 points were obtained for each recording. Since bright reflection spots arising from surface imperfections cannot be considered to be point sources (in contrast to the tracer particles), a lower accuracy for their triangulated position is expected and an accurate reconstruction of the 3D model surface was not deemed possible. As a consequence, the mean wall-normal location of the triangulated point cloud $\left(Y_{\text {ref }}^{*}\right)$ was used merely as a reference position for the instantaneous displacement of the wall surface along the wall normal direction. This position was used to correct the $Y^{*}$ location of the instantaneous tracks from MP-STB before performing the ensemble averaging in order to reduce the modulation effect introduced by the wall vibration. A maximum displacement of approximately $4 p x$ was found; based on the analysis of the standard deviation of the wall-normal position of the triangulated spots, an accuracy of approximately $35 \mu \mathrm{m}(1 p x)$ was estimated for the 

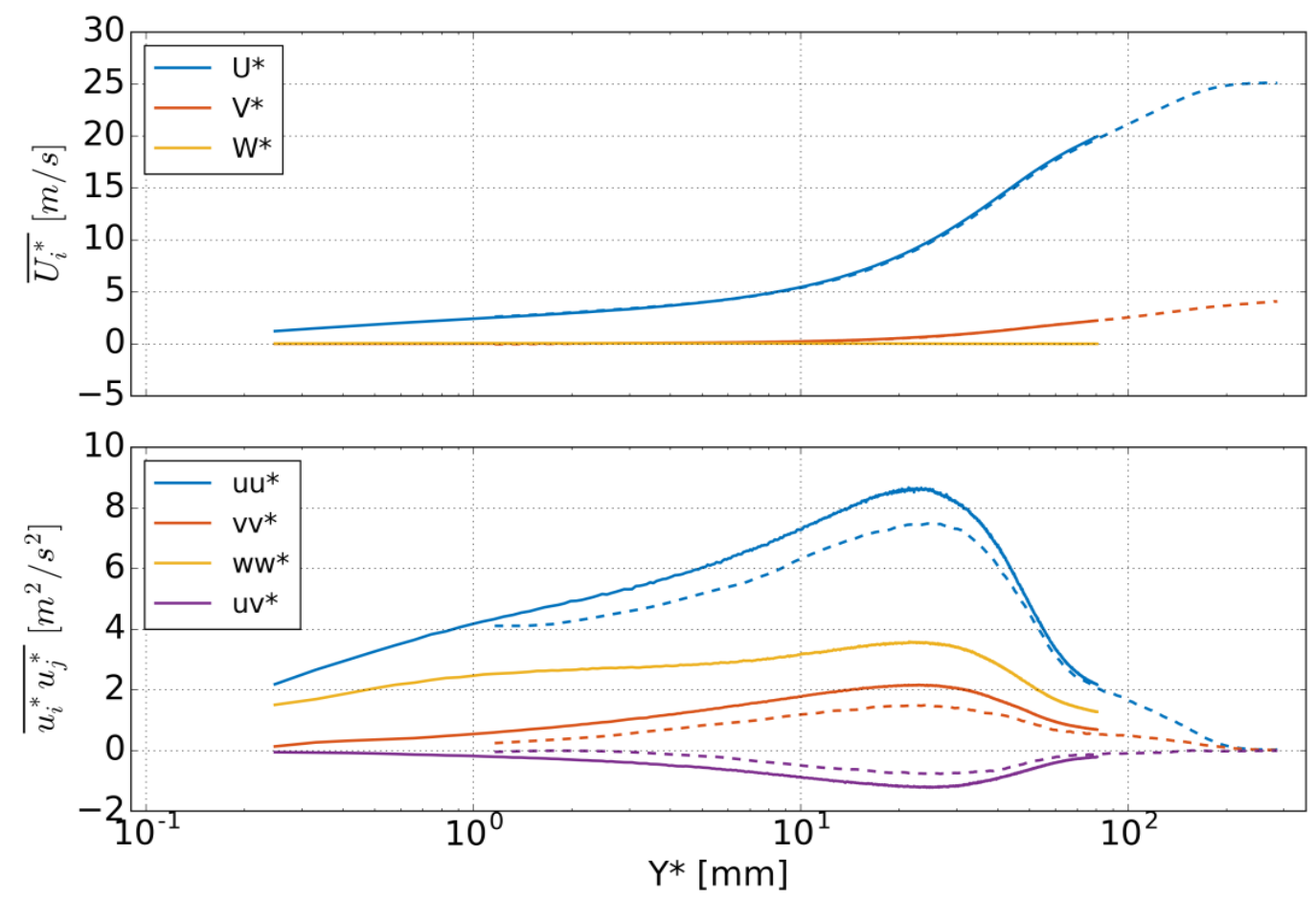

Figure 16. Mean velocity profiles and Reynolds stresses along the wall-normal direction at $X=-706 \mathrm{~mm}$; dashed and dotted lines for planar PIV and MP-STB results, respectively.

$Y_{r e f}^{*}$ location, effectively limiting the maximum spatial resolution (i.e. minimum size of the bins along the wallnormal direction) achievable by means of ensemble averaging of the track data.

A solution to this limitation could be the adoption of a glass or mirror-polished surface for the model; in this condition, the instantaneous wall position could be inferred by the triangulation of wall-particles (i.e. seeding droplets impacting and accumulating onto the surface). In the framework of a similar MP-STB investigation, Novara et al (2016a) demonstrated that the instantaneous location of the surface by means of triangulation of wall-particles, could be performed with a positional error lower than $0.1 \mathrm{px}$. This solution would also significantly reduce the magnitude of the reflection, allowing for the measurement of the near-wall region $\left(Y^{*}<0.1 \mathrm{~mm}\right)$. Due to practical limitations specific to the present experimental campaign, this solution could not be implemented and will be considered for future investigations.

The three velocity components $\left(U^{*}, V^{*}\right.$ and $\left.W^{*}\right)$ were extracted for each instantaneous track at the mid-point of the trajectory and collected over the recording sequence in two-dimensional bins of $450 \times 2 p x\left(16 \times 0.07 \mathrm{~mm}^{2}\right)$; approximately 100,000 individual samples from 40,000 independent four-pulse recordings were found within each bin and used to estimate the mean and fluctuating velocity components.

A coordinate transformation was applied to the results from the planar PIV analysis in order to obtain the mean and fluctuating velocity components along the wall-parallel and wall-normal directions. A direct comparison of the TBL profiles was carried out interpolating the PIV results onto the measured points from MP-STB at the center of the 3D 
field-of-view along the $Y^{*}$ axis $(X=-706 \mathrm{~mm})$; results are shown in Figure 16, concerning both the mean velocity components and the Reynolds stresses.

The maximum difference in terms of wind-tunnel velocity $\left(U_{e}\right)$ between the planar PIV and the MP-STB measurements was below 1\%. A good comparison between the mean velocity profiles from PIV and MP-STB is found for both the wall-parallel and wall-normal components; the maximum relative difference for the $\overline{U^{*}}$ component is of approximately $0.5 \%$ of $U_{e}$, Figure 16-top. The mean velocity profiles show the capability of the particle tracking based MP-STB technique to obtain valid measurement points in proximity of the wall (limited here to $0.2 \mathrm{~mm}$ from the model surface due to the strong laser light reflections), while the PIV method is limited by the finite size of the cross-correlation windows (approximately $1.6 \mathrm{~mm}$ in the wall-normal direction).

The wall-normal profiles relative to the Reynolds stresses are shown in in Figure 16-bottom; here the averaging effect of the large $24 \times 14 p x$ cross-correlation window (with respect to the $2 p x$ bins in wall-normal direction used for ensemble averaging of the tracking data) results in significant underestimation of the turbulent fluctuations of $10 \div 50 \%$ for the wall-parallel component. As expected, the PIV and tracking approaches deliver similar results in the outer region of the turbulent boundary layer $\left(Y^{*}>70 \mathrm{~mm}\right)$ where the spatial velocity gradients are less prominent. Results suggest the potential of the multi-exposed frame recording strategy to provide data suitable for accurate 3D Lagrangian particle tracking by means of Multi-Pulse STB, and confirm the capability of particle tracking approaches of delivering reliable measurements in close proximity of interfaces, walls and in strong shear layers (Kähler et al 2012a, 2012b).

\section{Conclusions and outlook}

The Multi-Pulse Shake-The-Box technique is applied to multi-pulse recordings from several experimental investigations of turbulent boundary layer flows in order to perform 3D Lagrangian particle tracking. The adoption of several acquisition strategies is discussed and assessed based on the performances of the MP-STB algorithm in terms of ratio of successfully reconstructed particle tracks.

Two pulse-separation strategies making use of a dual 3D imaging system, namely the polarization- and timing-based technique, have been employed for the investigation of a turbulent boundary layer flow at $10 \mathrm{~m} / \mathrm{s}$. Both techniques, in combination with the MP-STB method, delivered results that were found to be in good agreement with reference DNS data obtained at a similar Reynolds number. Nevertheless, the relatively poor performances in terms of track yield (approximately $60 \%$ and $70 \%$ for the polarization- and timing-based strategies respectively) suggest the possibility of loss-of-tracks due to the different viewing directions of the independent imaging systems with respect to the Mie scattering lobes from the particle tracers.

This issue, together with the need of simplifying the experimental setup and reducing the required optical access for industrial applications, led to the implementation of an acquisition strategy where a single multi-camera system is employed and the two camera frames are double-exposed to record the four pulses of the multi-pulse sequences.

The multi-exposed acquisition strategy is assessed here based on the MP-STB reconstruction of particle images from a turbulent boundary layer investigation at $15 \mathrm{~m} / \mathrm{s}$; results show that approximately $95 \%$ of the expected tracks can be successfully reconstructed. The artificial increase in the particle image density of double-exposed images poses a 
limit to the maximum applicable seeding concentration; nevertheless, data assimilation techniques such as FlowFit exploit physical constrains in order to increase the spatial resolution beyond the sampling offered by the scattered tracked particles. The instantaneous flow structures obtained with FlowFit interpolation are shown here in terms of iso-surfaces of $\mathrm{Q}$ criterion, allowing for the identification of vortical structures within the turbulent boundary layer flow. Furthermore, the instantaneous 3D pressure distribution can be recovered as part of the FlowFit data assimilation and interpolation.

Finally, a comparison between planar PIV and multi-exposed MP-STB in terms of flow statistics is presented based on the investigation of a turbulent boundary layer with adverse pressure gradient at approximately $30 \mathrm{~m} / \mathrm{s}$. As the two techniques have been applied to investigate the same region of the flow, a direct comparison of the mean velocity and turbulent fluctuations profiles is carried out. Results show the suitability of the MP-STB Lagrangian particle tracking technique in accurately capturing the turbulent fluctuations without introducing averaging effects typical of the cross-correlation based PIV approaches. Furthermore, as the average flow separation point approaches the MP-STB investigated volume, the capability of the tracking strategy in solving the flow direction ambiguity arising from the double-exposed frames is confirmed by the inspection of instantaneous 3D track fields.

Based on the experimental results shown in the present study, it can be concluded that, despite the increase in particle image density resulting from the double-exposed particle images, the adoption of multi-exposed recordings has the potential to become the technique of choice for the recording of multi-pulse sequences for Lagrangian particle tracking in high-speed flows. Besides the higher performances of the MP-STB in terms of track yield, this approach significantly simplifies the experimental setups by halving the number of required cameras, potentially promoting its use in industrial applications where the optical access to the investigated domain is typically limited.

A study on the limits of the MP-STB technique applied to multi-exposed recordings based on synthetic images can be foreseen and will be the subject of a future investigation. In such a work, the effect of several parameters (number of cameras, particle image density, temporal resolution and number of pulses - e.g. three-pulse STB) can be accurately assessed given the availability of the ground-truth solution. Furthermore, an alternative pulse distribution along the observation time can be envisioned, where the time separation between the pulses recorded on the same frame is increased, thus reducing the chance of overlapping particle images. Preliminary considerations regarding the measurement uncertainty have been presented here, which suggest a higher accuracy of this approach concerning the acceleration measurement. This strategy is currently being considered for upcoming multi-pulse 3D LPT investigations; the assessment of its performances in combination with the MP-STB algorithm is devoted to a future study.

\section{Acknowledgments}

The present work has been partially funded by the DFG-project "Analyse turbulenter Grenzschichten mit Druckgradient bei großen Reynolds-Zahlen mit hochauflösenden Vielkameramessverfahren". 


\section{References}

Adrian R (2007) “Hairpin vortex organization in wall turbulence" Physics of Fluids 19, 041301

Blinde P, Lynch KP, Schrijer F and van Oudheusden BW (2015) "Determination of instantaneous pressure in a transonic base flow using four-pulse tomographic PIV" $11^{\text {th }}$ International Symposium on PIV, 14-16 September, Santa Barbara, California, US

Cierpka C, Lütke B, Kähler CJ (2013) “Higher order multi-frame particle tracking velocimetry" Experiments In Fluids, 54, 1533

Elsinga GE, Scarano F, Wieneke B, van Oudheusden BW (2006) "Tomographic particle image velocimetry" Experiments In Fluids, 41, 933-947

Elsinga GE, Westerweel J, Scarano F and Novara M (2011) "On the velocity of ghost particles and the bias error in Tomographic-PIV” Experiments In Fluids, 50, 825-838

Geisler R (2014) “A fast double shutter system for CCD image sensors” Measurement Science and Technology, 25, 025404

Geisler R, Novara M and Schröder A (2016) "Volumetric Multi-Pulse Particle Tracking Measurement for Separated Laminar Transitional Flow Investigations” 18th Lisbon Symposium, July 4-7, Lisbon, Portugal

Gesemann S, Huhn F, Schanz D and Schröder A (2016) "From Noisy Particle Tracks to Velocity, Acceleration and Pressure Fields using B-splines and Penalties" 18th Lisbon Symposium, July 4-7, Lisbon, Portugal

Hain R, Kähler CJ (2007) “Fundamentals of multiframe particle image velocimetry (PIV)” Experiments In Fluids, $42,575-587$

Huhn F, Schanz D, Gesemann S, Dierksheide U, van de Meerendonk R and Schröder A (2017) "Large-scale volumetric flow measurement in a pure thermal plume by dense tracking of helium-filled soap bubbles" Experiments In Fluids, 58, 116

Huhn F, Schanz D, Manovski P, Gesemann S and Schröder A (2018) "Time-resolved large-scale volumetric pressure fields of an impinging jet from dense Lagrangian particle tracking” Experiments in Fluids, 59, 81

Jahn T (2017) "Volumetrische Strömungsvermessung: Eine Implementierung von Shake-The-Box", Masterarbeit, Georg-August-Universität Göttingen \& DLR Göttingen

Kähler CJ and Kompenhans J (2000) "Fundamentals of multiple plane stereo particle image velocimetry", Experiments in Fluids, 29, 70-77

Kähler CJ, Scharnowski S and Cierpka C (2012a) "On the uncertainty of digital PIV and PTV near walls" Experiments In Fluids, 52, 1641-1656 
Kähler CJ, Scharnowski S and Cierpka C (2012b) “On the resolution limit of digital PIV” Experiments In Fluids, 52, 1629-1639

Lynch KP and Scarano F (2013) "A high-order time-accurate interrogation method for time-resolved PIV" Measurement Science and Technology, 24, 035305

Lynch KP and Scarano F (2014) "Material acceleration estimation by four-pulse Tomo-PIV" Measurement Science and Technology, 25, 084005

Lynch KP and Scarano F (2015) "An efficient and accurate approach to MTE-MART for time-resolved tomographic PIV” Experiments In Fluids, 56, 66

Maas HG, Gruen A and Papantoniou D (1993) "Particle tracking velocimetry in three dimensional flows" Experiments In Fluids, 15, 133-146

Manovski P, Depuru Mohan NK, Geisler R, Novara M, Schanz D, Agocs J, Ahlefeldt T, Spehr C and Schröder A (2016) "3D Lagrangian particle tracking using 4-pulse Shake-The-Box synchronized with microphone measurements on a subsonic jet at Mach 0.9" NIOPLEX Workshop, October 25-26, Delft, The Netherlands

Michaelis D and Wolf CC (2011) "Vibration compensation for tomographic PIV using single image volume selfcalibration" $9^{\text {th }}$ International Symposium on PIV, July 21-23, Kobe, Japan

Novara M, Batenburg KJ and Scarano F (2010) "Motion tracking-enhanced MART for tomographic PIV" Measurement Science and Technology, 21, 035401

Novara M, Schanz D, Gesemann S, Lynch KP and Schröder A (2016b) "Lagrangian 3D particle tracking for multipulse systems: performance assessment and application of Shake-The-Box" 18th Lisbon Symposium, July 4-7, Lisbon, Portugal

Novara M, Schanz D, Reuther N, Kähler CJ and Schröder A (2016a) "Lagrangian 3D particle tracking in high-speed flows: Shake-The-Box for multi-pulse systems” Experiments In Fluids, 57, 128

Scarano F and Riethmuller ML (2000) "Advances in iterative multigrid PIV image processing" Experiments In Fluids, 51, 60

Scarano F and Westerweel J (2005) "Universal outlier detection for PIV data" Experiments In Fluids, 39, 6, 10961100

Schanz D, Gesemann S and Schröder A (2016) "Shake-The-Box: Lagrangian particle tracking at high particle image densities" Experiments In Fluids, 57, 70

Schanz D, Gesemann S, Schröder A, Wieneke B and Novara M (2013) "Non-uniform optical transfer function in particle imaging: calibration and application to tomographic reconstruction" Measurement Science and Technology, 24, 024009 
Schlatter P and Örlü R (2010) “Assessment of direct numerical simulation data of turbulent boundary layers" Journal of Fluid Mechanics, 659, 116-126

Schneiders JFG, Scarano F (2016) "Dense velocity reconstruction from tomographic PTV with material derivatives" Experiments In Fluids, 57, 139

Schröder A, Schanz D, Geisler R, Willert C and Michaelis D (2013) "Dual-volume and four-pulse Tomo-PIV using polarized light" $10^{\text {th }}$ International Symposium on PIV, July 1-3, Delft, The Netherlands

Schröder A, Schanz D, Novara M, Philipp F, Geisler R, Agocs J, Knopp T, Schroll M and Willert C (2018) "Investigation of a high Reynolds number turbulent boundary layer flow with adverse pressure gradients using PIV and 2D- and 3D- Shake-The-Box" $19^{\text {th }}$ Lisbon Symposium, July 16-19, Lisbon, Portugal

Sciacchitano A, Scarano F, Wieneke B (2012) "Multi-frame pyramid correlation for time-resolved PIV" Experiments In Fluids, 53, 1087-1105

Van Gent PL, Michaelis D, van Oudheusden BW, Weiss PE, de Kat R, Laskari A, Jeon YJ, David L, Schanz D, Huhn F, Gesemann S, Novara M, McPhaden C, Neeteson NJ, Rival DE, Schneiders JFG and Schrijer FFJ (2017) "Comparative assessment of pressure field reconstructions from particle image velocimetry measurements and Lagrangian particle tracking” Experiments In Fluids, 58, 33

Van Oudheusden BW (2013) "PIV-based pressure measurement" Measurement Science and Technology, 24, 032001

Wieneke B (2008) "Volume self-calibration for 3D particle image velocimetry" Experiments In Fluids, 45, 549-556

Wieneke B (2013) "Iterative reconstruction of volumetric particle distribution" Measurement Science and Technology, 24, 024008

Willert C "PIVview 2C/3C User Manual Version 2.3" http://www.pivtec.com 